\title{
Plasmon Mediated Energy Transport in PV Systems with Photo-Active Surface Modified Metallically in Nano-Scale and in Metallic Nano-Chains
}

\author{
Witold Jacak
}

Additional information is available at the end of the chapter

http://dx.doi.org/10.5772/50750

\section{Introduction}

Experimental and theoretical investigations of plasmon excitations in metallic nano-crystals rapidly grew up mainly due to possible applications in photo-voltaics and microelectronics. A significant enhancement of absorption of incident light in photo-diode-systems with active surfaces covered with nano-size metallic particles (of $\mathrm{Au}, \mathrm{Ag}$ or $\mathrm{Cu}$ ) with planar density $10^{8}-10^{10} / \mathrm{cm}^{2}$ was observed [24, 25, 27, 30, 32, 34]. These findings are of practical importance for enhancement of solar cell efficiency, especially for development of thin film cell technology. On the other hand, hybridized states of surface plasmons and photons result in plasmon-polaritons [20,36], which are of high importance for applications in photonics and microelectronics $[10,20]$, in particular, for sub-diffraction transportation of converted light energy and information in metallically modified structures in nano-scale [21,36].

Surface plasmons in nano-particles have been widely investigated since their classical description by Mie [23]. Many particular studies, including numerical modeling of multi-electron clusters, have been carried out [4,5]. They were mostly developments of Kohn-Sham attitude in form of LDA (Local Density Approximation) or TDLDA (Time Dependent LDA) for small metallic clusters only [4, 5, 8, 9, 17], up to ca 200 electrons (as limited by numerical calculation constraints that grow rapidly with the number of electrons). The random phase approximation (RPA) was formulated [28] for description of volume plasmons in bulk metals and utilized also for confined geometry mainly in a numerical or semi-numerical manner $[4,5,17]$. Usually, in these analyzes the jellium model was assumed for description of positive ion background in the metal and the dynamics was addressed to the electron system only $[5,8,17]$. Such an attitude is preferable for clusters of simple metals, including noble metals (also transition and alkali metals).

The classical Mie frequencies [23] are not dependent on the sphere radius, in contrast to the experimental observations for both small metallic clusters and larger spheres. The emerging 
of the Mie response from the more general behavior was presented [4, 5, 8]. Numerical analyzes (for clusters up to 200 electrons) revealed the red-shift of Mie resonance mainly due to so-called spill-out of the electron cloud beyond the jellium rim. This effect is, however, not important for particles with radii larger than $2 \mathrm{~nm}$, and for nanospheres, of size of $10-60 \mathrm{~nm}$, the other effects are responsible for size-dependent shifts of resonances, pronounced in large particles and opposite (with respect to the dependence versus $a$ ) to the red shift for small and ultra-small clusters.

The main factor for plasmon oscillations in large nanospheres was identified $[11,12,15]$ as radiation phenomena, which for radius larger than $10 \mathrm{~nm}$ dominate plasmon damping as growing with the radius $a$ as $a^{3}$. The pronounced cross-over in the vicinity of $10 \mathrm{~nm}$ (for Au, $\mathrm{Ag}$ and $\mathrm{Cu}$ ) has been predicted [15] — for smaller nanospheres, the scattering damping scaling with $a$ as $\frac{1}{a}$ (including inter-particle and boundary scattering and so-called Landau damping [35]) is important, while for larger spheres, the radiation losses are overwhelming and rapidly grow with $a$ as $a^{3}$. Thus rather large nanoparticles would play a role in phenomena linked with energy transport employing plasmon radiation, which well corresponds to experimental observations [26, 27, 30, 31, 33, 34].

Metallic nanospheres (or nanoparticles of other shape) can act as light converters, collecting energy of incident photons in surface plasmon oscillations. This energy can be next transferred to the semiconductor substrate in a more efficient manner in comparison to the direct photo-effect. Experimental observations [26, 27, 30, 31, 33, 34] suggest, that the near-field coupling between plasmons in nanospheres and band electrons in the semiconductor substrate allows for significant growth of selective light energy transformation into a photo-current in the diode system. This phenomenon is not described in detail as of yet and moreover, some competitive mechanisms apparently contribute. Nevertheless, one can argue generally, that due to the nano-scale size of the metallic components, the momentum is not conserved, which leads to the allowance of all indirect optical inter-band transitions in the semiconductor layer, resulting in enhancement of the photo-current in comparison to the ordinary photo-effect, when only direct inter-band transitions are admitted.

In the present paper we review the RPA (random phase approximation) semi-classical theory of plasmons, formulated [11] for large metallic nanospheres in analogy to RPA theory of plasmons in bulk metal [28, 29] (Pines-Bohm theory). The advantage of this description $[11,15]$ consists in full-analytic formulation allowing for application to more complicated physical situations, as e.g., plasmons interaction with surrounding medium, in order to describe plasmonic enhancement of photo-voltaic (PV) effect in the case of metallic particles deposited on the photo-active layer of the semiconductor, or for description of transport of plasmon-polaritons, with potential applications for sub-diffraction nano-photonics [6].

The coupling of surface plasmons in the near-field zone with another systems, like a semiconductor substrate or other metallic nanosphere in the chain, is the main topic of the present paper. An assessment of the energy transfer via this channel, as made in the present paper within the Fermi golden rule scheme, reveals a high efficiency of plasmon-mediated energy transport and explains the experimentally observed PV efficiency growth of solar cell setups with surface metallic nano-modifications. In seems to be of a particular significance for thin film semiconductor solar cell technology including conjugated polymer semiconductor photo-active matrices with a great potential commercial usage, providing an increase of their 
efficiency by relatively not-costly modifications, e.g., by sparse coverings of photo-active surface with noble metal nanoparticles, which is feasible in various techniques.

The paper is organized as follows. In the next section a short review of the model is given including results of analyzes both for volume and surface plasmon excitations in spherical geometry. Damping phenomena with particular role of radiation losses for large nanospheres is described in the following section, in terms of the Lorentz friction. The separate discussion is addressed to giant enhancement of photo-voltaic effect by plasmon mediation in near-field zone. The comparison with experimental data related to radiation of plasmons in metallic nanospheres is also given. In the last section, the undamped mode of collective surface plasmon excitation along the metallic nanosphere chain is demonstrated, including nonlinear effects.

\section{Surface and volume plasmons in large metallic nanospheres}

\subsection{RPA equation for collective electron oscillations in confined geometry}

The Hamiltonian for the metallic nanosphere has the form:

$$
\begin{aligned}
& \hat{H}=-\sum_{v=1}^{N} \frac{\hbar^{2} \nabla_{v}^{2}}{2 M}+\frac{1}{2} \sum_{v \neq v^{\prime}} u\left(\boldsymbol{R}_{v}-\boldsymbol{R}_{v^{\prime}}\right)-\sum_{j=1}^{N_{e}} \frac{\hbar^{2} \nabla_{j}^{2}}{2 m}+\frac{1}{2} \sum_{j \neq j^{\prime}} \frac{e^{2}}{\left|\boldsymbol{r}_{j}-\boldsymbol{r}_{j^{\prime}}\right|} \\
& +\sum_{v, j} w\left(\boldsymbol{R}_{v}-\boldsymbol{r}_{j}\right),
\end{aligned}
$$

where $\boldsymbol{R}_{v}, \boldsymbol{r}_{j}$ and $M, m$ are the positions and masses of the ions and electrons, respectively; $N$ is the number of ions in the sphere, $N_{e}=Z N$ is the number of collective electrons, $u\left(\boldsymbol{R}_{v}-\right.$ $\boldsymbol{R}_{v^{\prime}}$ ) is the interaction of ions (ion is treated as a nucleus with electron core of closed shells) and $w\left(\boldsymbol{R}_{v}-\boldsymbol{r}_{j}\right)$ is the local pseudopotential of electron-ion interaction. Assuming the jellium model $[3,5,7]$ one can write for the background ion charge uniformly distributed over the sphere: $n_{e}(\boldsymbol{r})=n_{e} \Theta(a-r)$, where $n_{e}=N_{e} / V$ and $n_{e}|e|$ is the averaged positive charge density, $V=\frac{4 \pi a^{3}}{3}$ is the sphere volume and $\Theta$ is the Heaviside step-function.

A local electron density can be written as follows [28, 29]:

$$
\rho(\boldsymbol{r}, t)=<\Psi_{e}(t)\left|\sum_{j} \delta\left(\boldsymbol{r}-\boldsymbol{r}_{j}\right)\right| \Psi_{e}(t)>
$$

with the Fourier picture:

$$
\tilde{\rho}(\boldsymbol{k}, t)=\int \rho(\boldsymbol{r}, t) e^{-i \boldsymbol{k} \cdot \boldsymbol{r}} d^{3} r=<\Psi_{e}(t)|\hat{\rho}(\boldsymbol{k})| \Psi_{e}(t)>,
$$

where the operator $\hat{\rho}(\boldsymbol{k})=\sum_{j} e^{-i \boldsymbol{k} \cdot \boldsymbol{r}_{j}}$.

Using the above notation one can rewrite the electron part of the Hamiltonian $(1), \hat{H}_{e}$, in the following form [11]:

$$
\begin{aligned}
& \hat{H}_{e}=\sum_{j=1}^{N_{e}}\left[-\frac{\hbar^{2} \nabla_{j}^{2}}{2 m}\right]-\frac{e^{2}}{(2 \pi)^{3}} \int d^{3} k \tilde{n}_{e}(\boldsymbol{k}) \frac{2 \pi}{k^{2}}\left(\hat{\rho^{+}}(\boldsymbol{k})+\hat{\rho}(\boldsymbol{k})\right) \\
& +\frac{e^{2}}{(2 \pi)^{3}} \int d^{3} k \frac{2 \pi}{k^{2}}\left[\hat{\rho^{+}}(\boldsymbol{k}) \hat{\rho}(\boldsymbol{k})-N_{e}\right],
\end{aligned}
$$


where: $\tilde{n}_{e}(\boldsymbol{k})=\int d^{3} r n_{e}(\boldsymbol{r}) e^{-i \boldsymbol{k} \cdot \boldsymbol{r}}, \frac{4 \pi}{k^{2}}=\int d^{3} r \frac{1}{r} e^{-i \boldsymbol{k} \cdot \boldsymbol{r}}$.

The motion equation has the form:

$$
\frac{d^{2} \hat{\rho}(\boldsymbol{k})}{d t^{2}}=\frac{1}{(i \hbar)^{2}}\left[\left[\hat{\rho}(\boldsymbol{k}), \hat{H}_{e}\right], \hat{H}_{e}\right] .
$$

Within the RPA and for Thomas-Fermi averaged kinetic energy formula [28], Eq. (5) attains the form [11]:

$$
\begin{aligned}
& \frac{\partial^{2} \delta \tilde{\rho}(\boldsymbol{r}, t)}{\partial t^{2}}=\left[\frac{2}{3} \frac{\epsilon_{F}}{m} \nabla^{2} \delta \tilde{\rho}(\boldsymbol{r}, t)-\omega_{p}^{2} \delta \tilde{\rho}(\boldsymbol{r}, t)\right] \Theta(a-r) \\
& -\frac{2}{3 m} \nabla\left\{\left[\frac{3}{5} \epsilon_{F} n_{e}+\epsilon_{F} \delta \tilde{\rho}(\boldsymbol{r}, t)\right] \frac{\boldsymbol{r}}{r} \delta(a-r)\right\} \\
& -\left[\frac{2}{3} \frac{\epsilon_{F}}{m} \frac{\boldsymbol{r}}{r} \nabla \delta \tilde{\rho}(\boldsymbol{r}, t)+\frac{\omega_{p}^{2}}{4 \pi} \frac{\boldsymbol{r}}{r} \nabla \int d^{3} r_{1} \frac{1}{\left|\boldsymbol{r}-\boldsymbol{r}_{1}\right|} \delta \tilde{\rho}\left(\boldsymbol{r}_{1}, t\right)\right] \delta(a-r) .
\end{aligned}
$$

In the above formula $\omega_{p}$ is the bulk plasmon frequency, $\omega_{p}^{2}=\frac{4 \pi n_{e} e^{2}}{m}$ (it was taken into account that $\left.\nabla \Theta(a-r)=-\frac{r}{r} \delta(a-r)\right)$. The solution of Eq. (6) can be decomposed into two parts related to the distinct domains:

$$
\delta \tilde{\rho}(\boldsymbol{r}, t)=\left\{\begin{array}{l}
\delta \tilde{\rho}_{1}(\boldsymbol{r}, t), \text { for } r<a, \\
\delta \tilde{\rho}_{2}(\boldsymbol{r}, t), \text { for } r \geq a,(r \rightarrow a+),
\end{array}\right.
$$

corresponding to the volume and surface excitations, respectively. These two parts of local electron density fluctuations satisfy the equations (according to Eq. (6)):

$$
\frac{\partial^{2} \delta \tilde{\rho}_{1}(\boldsymbol{r}, t)}{\partial t^{2}}=\frac{2}{3} \frac{\epsilon_{F}}{m} \nabla^{2} \delta \tilde{\rho}_{1}(\boldsymbol{r}, t)-\omega_{p}^{2} \delta \tilde{\rho}_{1}(\boldsymbol{r}, t),
$$

and (here $\epsilon=0+$ )

$$
\begin{aligned}
& \frac{\partial^{2} \delta \tilde{\rho}_{2}(\boldsymbol{r}, t)}{\partial t^{2}}=-\frac{2}{3 m} \nabla\left\{\left[\frac{3}{5} \epsilon_{F} n_{e}+\epsilon_{F} \delta \tilde{\rho}_{2}(\boldsymbol{r}, t)\right] \frac{\boldsymbol{r}}{r} \delta(a+\epsilon-r)\right\} \\
& -\left[\frac{2}{3} \frac{\epsilon_{F}}{m} \frac{\boldsymbol{r}}{r} \nabla \delta \tilde{\rho}_{2}(\boldsymbol{r}, t)+\frac{\omega_{p}^{2}}{4 \pi} \frac{\boldsymbol{r}}{r} \nabla \int d^{3} r_{1} \frac{1}{\left|\boldsymbol{r}-\boldsymbol{r}_{1}\right|}\left(\delta \tilde{\rho}_{1}\left(\boldsymbol{r}_{1}, t\right) \Theta\left(a-r_{1}\right)\right.\right. \\
& \left.\left.+\delta \tilde{\rho}_{2}\left(\boldsymbol{r}_{1}, t\right) \Theta\left(r_{1}-a\right)\right)\right] \delta(a+\epsilon-r) .
\end{aligned}
$$

\subsection{Solutions of plasmon RPA equations}

Eqs (8) and (9) can be solved upon imposed boundary and symmetry conditions. Let us represent both parts of the electron fluctuation in the following manner:

$$
\begin{aligned}
& \delta \tilde{\rho}_{1}(\boldsymbol{r}, t)=n_{e}\left[f_{1}(r)+F(\boldsymbol{r}, t)\right], \text { for } r<a, \\
& \delta \tilde{\rho}_{2}(\boldsymbol{r}, t)=n_{e} f_{2}(r)+\sigma(\Omega, t) \delta(r+\epsilon-a), \epsilon=0+, \text { for } r \geq a,(r \rightarrow a+),
\end{aligned}
$$

and now let us choose the convenient initial conditions, $\left.F(\boldsymbol{r}, t)\right|_{t=0}=0,\left.\sigma(\Omega, t)\right|_{t=0}=0$, ( $\Omega$ is the spherical angle), moreover $\left.\left(1+f_{1}(r)\right)\right|_{r=a}=\left.f_{2}(r)\right|_{r=a}$ (continuity condition), $\left.F(\boldsymbol{r}, t)\right|_{r \rightarrow a}=0, \int \rho(\boldsymbol{r}, t) d^{3} r=N_{e}$ (neutrality condition).

We arrive $[11,15]$ thus with the explicit form of the solutions of Eqs $(8,9)$ :

$$
\begin{aligned}
& f_{1}(r)=-\frac{k_{T} a+1}{2} e^{-k_{T}(a-r) \frac{1-e^{-2 k_{T} r}}{k_{T} r}}, \text { for } r<a, \\
& f_{2}(r)=\left[k_{T} a-\frac{k_{T} a+1}{2}\left(1-e^{-2 k_{T} a}\right)\right] \frac{e^{-k_{T}(r-a)}}{k_{T} r}, \text { for } r \geq a,
\end{aligned}
$$


where $k_{T}=\sqrt{\frac{6 \pi n_{e} e^{2}}{\epsilon_{F}}}=\sqrt{\frac{3 \omega_{p}^{2}}{v_{F}^{2}}}$ (Thomas-Fermi inverse radius). For the time-dependent parts of the electron fluctuations we find [11, 12]:

$$
F(\boldsymbol{r}, t)=\sum_{l=1}^{\infty} \sum_{m=-l}^{l} \sum_{n=1}^{\infty} A_{l m n} j_{l}\left(k_{n l} r\right) Y_{l m}(\Omega) \sin \left(\omega_{n l} t\right),
$$

and

$$
\begin{aligned}
& \sigma(\Omega, t)=\sum_{l=1}^{\infty} \sum_{m=-l}^{l} \frac{B_{l m}}{a^{2}} Y_{l m}(\Omega) \sin \left(\omega_{0 l} t\right) \\
& +\sum_{l=1}^{\infty} \sum_{m=-l}^{l} \sum_{n=1}^{\infty} A_{l m n} \frac{(l+1) \omega_{p}^{2}}{l \omega_{p}^{2}-(2 l+1) \omega_{n l}^{2}} Y_{l m}(\Omega) n_{e} \int_{0}^{a} d r_{1} \frac{r_{1}^{l+2}}{a^{l+2}} j_{l}\left(k_{n l} r_{1}\right) \sin \left(\omega_{n l} t\right),
\end{aligned}
$$

where $j_{l}(\xi)=\sqrt{\frac{\pi}{2 \xi}} I_{l+1 / 2}(\xi)$ is the spherical Bessel function, $Y_{l m}(\Omega)$ is the spherical function, $\omega_{n l}=\omega_{p} \sqrt{1+\frac{x_{n l}^{2}}{k_{T}^{2} a^{2}}}$ are the frequencies of electron volume self-oscillations (volume plasmon frequencies), $x_{n l}$ are the nodes of the Bessel function $j_{l}(\xi), k_{n l}=x_{n l} / a, \omega_{0 l}=\omega_{p} \sqrt{\frac{l}{2 l+1}}$ are the frequencies of electron surface self-oscillations (surface plasmon frequencies).

From the above equations it follows thus that the local electron density (within RPA attitude) has the form:

$$
\rho(\boldsymbol{r}, t)=\rho_{0}(r)+\rho_{\text {neq }}(\boldsymbol{r}, t),
$$

where the RPA equilibrium electron distribution is (correcting the uniform distribution $n_{e}$ ):

$$
\rho_{0}(r)=\left\{\begin{array}{l}
n_{e}\left[1+f_{1}(r)\right], \text { for } r<a, \\
n_{e} f_{2}(r), \text { for } r \geq a, r \rightarrow a+
\end{array}\right.
$$

and the nonequilibrium, of plasmon oscillation type, is:

$$
\rho_{\text {neq }}(\boldsymbol{r}, t)=\left\{\begin{array}{l}
n_{e} F(\boldsymbol{r}, t), \text { for } r<a, \\
\sigma(\Omega, t) \delta(a+\epsilon-r), \epsilon=0+, \text { for } r \geq a, r \rightarrow a+.
\end{array}\right.
$$

The function $F(\boldsymbol{r}, t)$ displays volume plasmon oscillations, while $\sigma(\Omega, t)$ describes the surface plasmon oscillations. Let us emphasize that in the formula for $\sigma(\Omega, t)$, Eq. (13), the first term corresponds to surface self-oscillations, while the second term describes the surface oscillations induced by the volume plasmons. The frequencies of the surface self-oscillations are

$$
\omega_{0 l}=\omega_{p} \sqrt{\frac{l}{2 l+1}}
$$

which, for $l=1$, agrees with the dipole type surface oscillations described originally by Mie [23], $\omega_{01}=\omega_{p} / \sqrt{3}$ (for simplicity, denoted hereafter as $\omega_{1}=\omega_{01}$ ).

\section{Damping of plasmons in large nanospheres}

One can phenomenologically include damping of plasmons in analogy to oscillator damping via the additional term, $-\frac{2}{\tau_{0}} \frac{\partial \delta \rho(\boldsymbol{r}, t)}{\partial t}$, to the right-hand-side of plasmon dynamic equations. They attain the form:

$$
\frac{\partial^{2} \delta \rho_{1}(\boldsymbol{r}, t)}{\partial t^{2}}+\frac{2}{\tau_{0}} \frac{\partial \delta \rho_{1}(\boldsymbol{r}, t)}{\partial t}=\frac{2}{3} \frac{\epsilon_{F}}{m} \nabla^{2} \delta \rho_{1}(\boldsymbol{r}, t)-\omega_{p}^{2} \delta \rho_{1}(\boldsymbol{r}, t),
$$


and

$$
\begin{aligned}
& \frac{\partial^{2} \delta \rho_{2}(\boldsymbol{r}, t)}{\partial t^{2}}+\frac{2}{\tau_{0}} \frac{\partial \delta \rho_{2}(\boldsymbol{r}, t)}{\partial t}=-\frac{2}{3 m} \nabla\left\{\left[\frac{3}{5} \epsilon_{F} n_{e}+\epsilon_{F} \delta \rho_{2}(\boldsymbol{r}, t)\right] \frac{\boldsymbol{r}}{r} \delta(a+\epsilon-r)\right\} \\
& -\left[\frac{2}{3} \frac{\epsilon_{F}}{m} \frac{\boldsymbol{r}}{r} \nabla \delta \rho_{2}(\boldsymbol{r}, t)+\frac{\omega_{p}^{2}}{4 \pi} \frac{r}{r} \nabla \int d^{3} r_{1} \frac{1}{\left|\boldsymbol{r}-\boldsymbol{r}_{1}\right|}\left(\delta \rho_{1}\left(\boldsymbol{r}_{1}, t\right) \Theta\left(a-r_{1}\right)\right.\right. \\
& \left.\left.+\frac{1}{\varepsilon} \delta \rho_{2}\left(\boldsymbol{r}_{1}, t\right) \Theta\left(r_{1}-a\right)\right)+\frac{e n_{e}}{m} \frac{\boldsymbol{r}}{r} \cdot \boldsymbol{E}(t)\right] \delta(a+\epsilon-r) .
\end{aligned}
$$

For the homogeneous forcing field $\boldsymbol{E}(t)$ (this corresponds to dipole approximation satisfied for $a \sim 10-50 \mathrm{~nm}$, when $\lambda \sim 500 \mathrm{~nm}$ ), only dipole surface mode can be excited and the electron dynamics resolves to the equation for a single dipole type mode, described by the function $Q_{1 m}(t)$. The function $Q_{1 m}(t)$ satisfies the equation:

$$
\begin{aligned}
& \frac{\partial^{2} Q_{1 m}(t)}{\partial t^{2}}+\frac{2}{\tau_{0}} \frac{\partial Q_{1 m}(t)}{\partial t}+\omega_{1}^{2} Q_{1 m}(t) \\
& =\sqrt{\frac{4 \pi}{3}} \frac{e n_{e}}{m}\left[E_{z}(t) \delta_{m 0}+\sqrt{2}\left(E_{x}(t) \delta_{m 1}+E_{y}(t) \delta_{m-1}\right)\right],
\end{aligned}
$$

where $\omega_{1}=\omega_{01}=\frac{\omega_{p}}{\sqrt{3 \varepsilon}}$ (it is a dipole-type surface plasmon Mie frequency [23]). Only this function contributes to the dynamical response to the homogeneous electric field. Thus for the homogeneous forcing field, electron density fluctuations:

$$
\delta \rho(\boldsymbol{r}, t)=\left\{\begin{array}{l}
0, \text { for } r<a, \\
\sum_{m=-1}^{1} Q_{1 m}(t) Y_{1 m}(\Omega) \text { for } r \geq a, r \rightarrow a+.
\end{array}\right.
$$

For plasmon oscillations given by Eq. (21) one can calculate the corresponding dipole,

$$
\boldsymbol{D}(t)=e \int d^{3} r \boldsymbol{r} \delta \rho(\boldsymbol{r}, t)=\frac{4 \pi}{3} e \boldsymbol{q}(t) a^{3},
$$

where $Q_{11}(t)=\sqrt{\frac{8 \pi}{3}} q_{x}(t), Q_{1-1}(t)=\sqrt{\frac{8 \pi}{3}} q_{y}(t), Q_{10}(t)=\sqrt{\frac{4 \pi}{3}} q_{x}(t)$ and $\boldsymbol{q}(t)$ satisfies the equation (cf. Eq. (20)),

$$
\left[\frac{\partial^{2}}{\partial t^{2}}+\frac{2}{\tau_{0}} \frac{\partial}{\partial t}+\omega_{1}^{2}\right] \boldsymbol{q}(t)=\frac{e n_{e}}{m} \boldsymbol{E}(t) .
$$

There are various mechanisms of plasmon damping, which could be effectively accounted for via phenomenological oscillator type damping term. All types of scattering phenomena, including electron-electron and electron-phonon interactions, as well contribution of boundary scattering effect [6] cause significant attenuation of plasmons, in particular, in small metal clusters. All these contributions to damping time ratio scale as $\frac{1}{a}$ and are of lowering significance with the radius growth. In the following subsection we argue that damping of plasmons caused by radiation losses scales conversely, as $a^{3}$, and for large nanospheres this channel dominates plasmon attenuation.

\subsection{Lorentz friction for plasmons}

The nanosphere surface plasmons can be induced by a homogeneous electric field [15], while the volume mode excitations need field inhomogeneity on the radius scale (and therefore the visible light cannot excite volume modes in the nanospheres with radii of $10-50 \mathrm{~nm}$ ). Plasmon oscillations are themselves a source of the e-m radiation. This radiation takes away the energy of plasmons resulting in their damping, which can be described as the 
Lorentz friction [18]. This damping was not included in $\tau_{0}$ in Eq. (23). The latter accounted for scattering of electrons on other electrons, on defects, on phonons and on nanoparticle boundary-all they lead to damping rate expressed by the simplified formula [6]:

$$
\frac{1}{\tau_{0}} \simeq \frac{v_{F}}{2 \lambda_{B}}+\frac{c v_{F}}{2 a}
$$

where, $C$ is the constant of unity order, $a$ is the nanosphere radius, $v_{F}$ is the Fermi velocity in metal, $\lambda_{B}$ is the electron free path in bulk (including scattering of electrons on other electrons, on impurities and on phonons [6]); for $\mathrm{Ag}, v_{F}=1.4 \times 10^{6} \mathrm{~m} / \mathrm{s}$ and $\lambda_{B} \simeq 57 \mathrm{~nm}$ (at room temperature); the latter term in the formula (24) accounts for scattering of electrons on the boundary of the nanoparticle, while the former one corresponds to scattering processes similar as in bulk. The other effects, as the so-called Landau damping (especially important in small clusters $[9,35])$, corresponding to decay of plasmon for high energy particle-hole pair, are of lowering significance for nanosphere radii larger than $2-3 \mathrm{~nm}$ [35] and are completely negligible for radii larger than $10 \mathrm{~nm}$. Note that the similarly lowering role with the radius growth plays also electron liquid spill-out effect $[5,8]$, though it was of primary importance for small clusters $[5,17]$.

The e-m wave emission caused electron friction can be described as the additional electric field [18],

$$
\boldsymbol{E}_{L}=\frac{2}{3 \varepsilon^{3 / 2} v^{3}} \frac{\partial^{3} \boldsymbol{D}(t)}{\partial t^{3}}
$$

where $v=\frac{c}{\sqrt{\varepsilon}}$ is the light velocity in the dielectric medium, and $\boldsymbol{D}(t)$ is the dipole of the nanosphere. According to Eq. (22) we arrive at the following:

$$
\boldsymbol{E}_{L}=\frac{2 e}{3 \varepsilon v^{2}} \frac{4 \pi}{3} a^{3} \frac{\partial^{3} \boldsymbol{q}(t)}{\partial t^{3}} .
$$

Substituting this into Eq. (23), we get

$$
\left[\frac{\partial^{2}}{\partial t^{2}}+\frac{2}{\tau_{0}} \frac{\partial}{\partial t}+\omega_{1}^{2}\right] \boldsymbol{q}(t)=\frac{e n_{e}}{m} \boldsymbol{E}(t)+\frac{2}{3 \omega_{1}}\left(\frac{\omega_{1} a}{v}\right)^{3} \frac{\partial^{3} \boldsymbol{q}(t)}{\partial t^{3}} .
$$

If one rewrites the above equation (for $\boldsymbol{E}=0$ ) in the form

$$
\left[\frac{\partial^{2}}{\partial t^{2}}+\omega_{1}^{2}\right] \boldsymbol{q}(t)=\frac{\partial}{\partial t}\left[-\frac{2}{\tau_{0}}+\frac{2}{3 \omega_{1}}\left(\frac{\omega_{1} a}{v}\right)^{3} \frac{\partial^{2} \boldsymbol{q}(t)}{\partial t^{2}}\right]
$$

thus, one notes that the zeroth order approximation (neglecting attenuation) corresponds to the equation:

$$
\left[\frac{\partial^{2}}{\partial t^{2}}+\omega_{1}^{2}\right] \boldsymbol{q}(t)=0
$$

In order to solve Eq. (28) in the next step of perturbation iteration, one can substitute, in the right-hand-side of this equation, $\frac{\partial^{2} \boldsymbol{q}(t)}{\partial t^{2}}$ by $-\omega_{1}^{2} \boldsymbol{q}(t)$ (acc. to Eq. (29)).

Therefore, if one assumes the above estimation, $\frac{\partial^{3} \boldsymbol{q}(t)}{\partial t^{3}} \simeq-\omega_{1}^{2} \frac{\partial \boldsymbol{q}(t)}{\partial t}$, one can include the Lorentz friction in a renormalized damping term:

$$
\left[\frac{\partial^{2}}{\partial t^{2}}+\frac{2}{\tau} \frac{\partial}{\partial t}+\omega_{1}^{2}\right] \boldsymbol{q}(t)=\frac{e n_{e}}{m} \boldsymbol{E}(t),
$$


where

$$
\frac{1}{\tau}=\frac{1}{\tau_{0}}+\frac{\omega_{1}}{3}\left(\frac{\omega_{1} a}{v}\right)^{3} \simeq \frac{v_{F}}{2 \lambda_{B}}+\frac{C v_{F}}{2 a}+\frac{\omega_{1}}{3}\left(\frac{\omega_{1} a}{v}\right)^{3},
$$

where we used for $\frac{1}{\tau_{0}} \simeq \frac{v_{F}}{2 \lambda_{B}}+\frac{C v_{F}}{2 a}$ [6]. Renormalized damping causes a change in the shift of self-frequencies of free surface plasmons, $\omega_{1}^{\prime}=\sqrt{\omega_{1}^{2}-\frac{1}{\tau^{2}}}$.

Note also, that one can verify [15] the above calculated Lorentz friction contribution to plasmon damping by estimation of the energy transfer in the far-field zone (which can be expressed by the Poynting vector) and via comparison with the energy loss of plasmon oscillation. We have arrived $[11,15]$ at the same formula for damping time rate as given by Eq. (30). The radius dependent shift of the resonance resulting due to strong irradiation-induced plasmon damping was verified also experimentally [15] by measurement of light extinction in colloidal solutions of nanoparticles with different size (it is done [15] for $\mathrm{Au}, 10-80 \mathrm{~nm}$, and $\mathrm{Ag}, 10-60 \mathrm{~nm}$ ). These measurements clearly support the $a^{3}$ plasmon damping behavior, as described above for the far-field zone radiation losses in a dielectric surroundings.

If, however, in the vicinity of the nanosphere the another system is located, the situation would change. For the case when the nanosphere is deposited on the semiconductor surface, the near-field coupling of plasmons with semiconductor band electrons must be included.

\section{Mediating of light energy transfer by surface plasmons in near-field regime}

Let us calculate the probability (per time unit) of inter-band transitions of electrons in substrate semiconductor covered with metallic nanospheres (with radius $a$ ), induced by photon-excited surface plasmons in metallic components. These plasmons excited in nanospheres deposited on the semiconductor layer, couple to the semiconductor band electrons in the near-field regime. The situation is similar to the ordinary photo-effect, though the perturbation of the electron system in semiconductor is not of a plane-wave form as it was in the case of direct illumination of semiconductor by incident photons, but attains the form of dipole-type near-field electric interaction [11,18]. This causes a change of the matrix element in the relevant Fermi golden formula. The potential for the near-field interaction of the surface Mie type plasmons with the band electrons can be written as [18]:

$$
\begin{aligned}
& w=e \psi(\mathbf{R}, t)=\frac{e}{\varepsilon_{0} R^{2}} \hat{\mathbf{n}} \cdot \mathbf{D}_{0} \sin (\omega t+\alpha)=w^{+} e^{i \omega t}+w^{-} e^{-i \omega t}, \\
& w^{+}=\left(w^{-}\right)^{*}=\frac{e}{\varepsilon_{0} R^{2}} \frac{e^{i \alpha}}{2 i} \hat{\mathbf{n}} \cdot \mathbf{D}_{0} .
\end{aligned}
$$

The terms $w^{+}, w^{-}$correspond to emission and absorption, respectively ( $\hat{\mathbf{n}}=\frac{\mathbf{R}}{R}, \mathbf{D}_{0}$ is the dipole plasmon amplitude). We choose the first one in order to consider emission of energy from the plasmon oscillations and transfer of it to the electron system in the semiconductor substrate. The semiconductor band system we model in the simplest single-band parabolic effective mass approximation. The inter-band transition probability is given by the Fermi golden rule,

$$
w\left(\mathbf{k}_{1}, \mathbf{k}_{2}\right)=\frac{2 \pi}{\hbar}\left|<\mathbf{k}_{1}\right| w^{+}\left|\mathbf{k}_{2}>\right|^{2} \delta\left(E\left(\mathbf{k}_{1}\right)-E\left(\mathbf{k}_{2}\right)+\hbar \omega\right),
$$


where Bloch states in the conduction and valence bands we assume as planar waves (for the sake of simplicity),

$$
\begin{aligned}
& \Psi_{\mathbf{k}_{1}}=\frac{1}{(2 \pi)^{3 / 2}} e^{i \mathbf{k}_{1} \cdot \mathbf{R}-i E\left(\mathbf{k}_{1}\right) t / \hbar}, \Psi_{\mathbf{k}_{2}}=\frac{1}{(2 \pi)^{3 / 2}} e^{i \mathbf{k}_{2} \cdot \mathbf{R}-i E\left(\mathbf{k}_{2}\right) t / \hbar}, \\
& E\left(\mathbf{k}_{1}\right)=-\frac{\hbar^{2} \mathbf{k}_{1}^{2}}{2 m_{p}^{*}}-E_{g}, E\left(\mathbf{k}_{2}\right)=\frac{\hbar^{2} \mathbf{k}_{2}^{2}}{2 m_{n}^{*}}
\end{aligned}
$$

(indices $n, p$ refer to electrons from the conduction and valence bands, respectively, $E_{g}$ is the forbidden gap).

The electron wave functions are normalized to the Dirac delta, which corresponds to infinite movement and the continuous energy spectrum. The wave function modulus squares do not have, in this case, probability interpretation (which must be normalized to the unity), therefore the expression, $w\left(\mathbf{k}_{1}, \mathbf{k}_{2}\right)=\frac{2 \pi}{\hbar}\left|<\mathbf{k}_{1}\right| w^{+}\left|\mathbf{k}_{2}>\right|^{2} \delta\left(E\left(\mathbf{k}_{1}\right)-E\left(\mathbf{k}_{2}\right)+\hbar \omega\right)$, has to be divided by the delta Dirac square, i.e., by the factor, $\left(\frac{V}{(2 \pi)^{3}}\right)^{2}$. This factor corresponds to the probability proper normalization, because,

$$
\frac{1}{(2 \pi)^{3}} \int d^{3} r e^{i \mathbf{k} \cdot \mathbf{r}}=\delta(\mathbf{k}) \simeq(\text { for } \mathbf{k}=0) \frac{V}{(2 \pi)^{3}}(V \rightarrow \infty)
$$

Note, that the same factor occurs also due to density of states, when one integrates over all initial and final states $\mathbf{k}_{1}, \mathbf{k}_{2}$. Two integrals will give the factor $\left(\frac{2 V}{(2 \pi)^{3}}\right)^{2},(2$ is caused by spin degeneration). Thus, both renormalized factors cancel mutually themselves.

Taking into account the above described renormalization, one can find the matrix element,

$$
<\mathbf{k}_{1}\left|w^{+}\right| \mathbf{k}_{2}>=\frac{1}{(2 \pi)^{3}} \int d^{3} R \frac{e}{\varepsilon_{0} 2 i} e^{i \alpha} \hat{\mathbf{n}} \cdot \mathbf{D}_{0} \frac{1}{R^{2}} e^{-i\left(\mathbf{k}_{1}-\mathbf{k}_{2}\right) \cdot \mathbf{R}} .
$$

Let us introduce the vector $\mathbf{q}=\mathbf{k}_{2}-\mathbf{k}_{1}$. One can choose the coordinate system in such a way, that the vector $\mathbf{q}$ is oriented along the $z$ axis, and the vector $\mathbf{D}_{0}$ lies in the plane $z x$ (as is depicted in the Fig. 1). Then, $\mathbf{q}=(0,0, q), \hat{\mathbf{n}}=\frac{\mathbf{R}}{R}=\left(\sin \Theta_{1} \cos \psi_{1}, \sin \Theta_{1} \sin \psi_{1}, \cos \Theta_{1}\right), \mathbf{R}=$ $R\left(\sin \Theta_{1} \cos \psi_{1}, \sin \Theta_{1} \sin \psi_{1}, \cos \Theta_{1}\right), \mathbf{D}_{0}=D_{0}(\sin \Theta, 0, \cos \Theta)$ and

$$
\begin{gathered}
\mathbf{q} \cdot \mathbf{R}=q R \cos \Theta_{1}, \\
\hat{\mathbf{n}} \cdot \mathbf{D}_{0}=D_{0}\left(\sin \Theta \sin \Theta_{1} \cos \psi_{1}+\cos \Theta \cos \Theta_{1}\right) .
\end{gathered}
$$

Hence,

$$
\begin{aligned}
& <\mathbf{k}_{1}\left|w^{+}\right| \mathbf{k}_{2}> \\
& =\frac{1}{(2 \pi)^{3}} \int d^{3} R \frac{e}{\varepsilon_{0} 2 i} e^{i \alpha} \hat{\mathbf{n}} \cdot \mathbf{D}_{0} \frac{1}{R^{2}} e^{-i\left(\mathbf{k}_{1}-\mathbf{k}_{2}\right) \cdot \mathbf{R}} \\
& =\frac{1}{(2 \pi)^{3}} \frac{e e^{i \alpha}}{\varepsilon_{0} 2 i} D_{0} \int_{a}^{\infty} \frac{R^{2}}{R^{2}} d R \int_{0}^{\pi} \\
& \times \sin \Theta_{1} d \Theta_{1} \int_{0}^{2 \pi} d \psi_{1}\left\{\cos \Theta \cos \Theta_{1}+\sin \Theta \sin \Theta_{1} \cos \psi_{1}\right\} e^{i q R \cos \Theta_{1}} \\
& =\frac{1}{(2 \pi)^{3}} \frac{e e^{i \alpha}}{\varepsilon_{0} 2 i} D_{0} \cos \Theta 2 \pi \int_{a}^{\infty} d R \int_{0}^{\pi} \cos \Theta_{1} \sin \Theta_{1} d \Theta_{1} e^{i q R \cos \Theta_{1}},
\end{aligned}
$$

the integer over $d \psi_{1}$ vanishes the second term in the parenthesis and only the first term contributes with the factor $2 \pi$. Note that,

$$
\int_{0}^{\pi} \cos \Theta_{1} \sin \Theta_{1} d \Theta_{1} e^{i x \cos \Theta_{1}}=-i \frac{d}{d x} \int_{0}^{\pi} \sin \Theta_{1} e^{i x \cos \Theta_{1}}=-i \frac{d}{d x} 2 \frac{\sin x}{x} .
$$




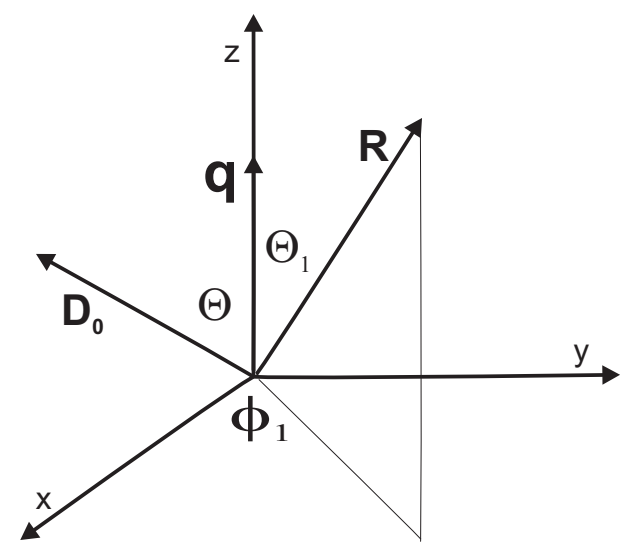

Figure 1. The reference frame is chosen in the way that the vector $\mathbf{q}$ is oriented along the axis $z$, while the vector $\mathbf{D}_{0}$ lies in the plane $x z$

Thus,

$$
\begin{aligned}
& <\mathbf{k}_{1}\left|w^{+}\right| \mathbf{k}_{2}>=\frac{-1}{(2 \pi)^{3}} \frac{e e^{i \alpha}}{\varepsilon_{0}} D_{0} \cos \Theta(2 \pi) \int_{a}^{\infty} d R \frac{1}{q} \frac{d}{d R} \frac{\sin q R}{q R} \\
& =\frac{1}{(2 \pi)^{2}} \frac{e e^{i \alpha}}{\varepsilon_{0}} D_{0} \cos \Theta \frac{1}{q} \frac{\sin q a}{q a} \rightarrow_{a \rightarrow 0} \frac{1}{(2 \pi)^{2}} \frac{e e^{i \alpha}}{\varepsilon_{0}} D_{0} \cos \Theta \frac{1}{q} .
\end{aligned}
$$

The lower limit of integral with respect to $R$ is taken as $a$ (the nanosphere radius). In this manner one can limit the accessible space for planar waves in the semiconductor, if assumes, for a model, a completely embedded nanosphere in surrounding material. This is, however, a limiting approximation as tunneling effect certainly would allow an access to the inner space, to some extent, at least. In order to account for that the nanosphere is deposited on the semiconductor surface we will confine integration with respect to $d \Theta_{1}$ to the segment $[0, \pi / 2]$, instead of $[0, \pi]$. We obtain in this case the following matrix element,

$$
\begin{aligned}
& <\mathbf{k}_{1}\left|w^{+}\right| \mathbf{k}_{2}>=\frac{1}{(2 \pi)^{3}} \frac{e e^{i \alpha}}{2 i \varepsilon_{0}} D_{0} \cos \Theta(2 \pi) \int_{a}^{\infty} d R \frac{1}{q} \frac{d}{d R} \frac{1-e^{i q R}}{q R} \\
& =\frac{1}{(2 \pi)^{2}} \frac{e e^{i \alpha}}{2 i \varepsilon_{0}} D_{0} \cos \Theta \frac{1}{q} \frac{1-e^{i q a}}{q a} \rightarrow_{a \rightarrow 0} \frac{1}{(2 \pi)^{2}} \frac{e e^{i \alpha}}{2 i \varepsilon_{0}} D_{0} \cos \Theta \frac{1}{q},
\end{aligned}
$$

because,

$$
\int_{0}^{\pi / 2} \cos \Theta_{1} \sin \Theta_{1} d \Theta_{1} e^{i x \cos \Theta_{1}}=-i \frac{d}{d x} \int_{0}^{\pi / 2} \sin \Theta_{1} e^{i x \cos \Theta_{1}}=\frac{d}{d x} \frac{1-e^{i x}}{x} .
$$

In the limit $a=0$, the modulus of the matrix element will diminish twice in comparison to the case when integration over $d \Theta_{1}$ had been taken over the whole space.

Now we will integrate over all initial and final states of both bands. The related integration over $\mathbf{k}_{1}, \mathbf{k}_{2}$, one can substitute with integration over $\mathbf{q}, \mathbf{k}_{2}$. Scalar products are invariant against coordinate systems rotations, therefore the result of integration will be the same if $\mathbf{q}$ was along $z$ axis or $\mathbf{D}_{0}$ is oriented now along the $z$ axis $-\Theta$ gives, in the latter case, the deviation of $\mathbf{q}$ with respect to $z$ direction - this choice of the reference frame is convenient for integration with respect to $d \mathbf{q}$.

Thus we arrive with the formula for transition probability in the following form,

$$
\delta w=\int d^{3} k_{1} \int d^{3} k_{2}\left[f_{1}\left(1-f_{2}\right) w\left(\mathbf{k}_{1}, \mathbf{k}_{2}\right)-f_{2}\left(1-f_{1}\right) w\left(\mathbf{k}_{2}, \mathbf{k}_{1}\right)\right],
$$


where, $f_{1}, f_{2}$ assign the temperature dependent distribution functions (Fermi-Dirac distribution functions) for initial and final states, respectively. The emission and absorption were included, but for room temperatures one can assume, $f_{2} \simeq 0$ and $f_{1} \simeq 1$, which leads to,

$$
\delta w=\int d^{3} k_{1} \int d^{3} k_{2} w\left(\mathbf{k}_{1}, \mathbf{k}_{2}\right) .
$$

In the above formula we avoided the density state factors canceled by probability renormalization, as was mentioned above.

We have to calculate the following integral,

$$
\delta w=\int d^{3} k_{2} \int d^{3} q \frac{e^{2}}{(2 \pi)^{3} \hbar \varepsilon_{0}} \frac{D_{0}^{2} \cos ^{2} \Theta}{q^{2}} \frac{\sin ^{2}(q a)}{(q a)^{2}} \delta\left(\frac{\hbar^{2} k_{1}^{2}}{2 m_{n}^{*}}+\frac{\hbar^{2} k_{2}}{2 m_{p}^{*}}-\left(\hbar \omega-E_{g}\right)\right),
$$

with $\delta\left(\frac{\hbar^{2} k_{1}^{2}}{2 m_{n}^{*}}+\frac{\hbar^{2} k_{2}}{2 m_{p}^{*}}-\left(\hbar \omega-E_{g}\right)\right)=\frac{1}{\alpha+\beta} \frac{1}{2 \beta^{\prime} q k_{2}} \delta\left(\cos \Theta_{2}-\frac{k_{2}^{2}+\beta^{\prime} q^{2}-\gamma^{\prime}}{2 \beta^{\prime} q k_{2}}\right)$, where, $\alpha=\frac{\hbar^{2}}{2 m_{n}^{*}}, \beta=$ $\frac{\hbar^{2}}{2 m_{p}^{*}}, \gamma=\hbar \omega-E_{g}, \alpha^{\prime}=\frac{\alpha}{\alpha+\beta}, \beta^{\prime}=\frac{\beta}{\alpha+\beta}, \gamma^{\prime}=\frac{\gamma}{\alpha+\beta}$. For each integral, with respect to $\mathbf{k}_{2}$ and $\mathbf{q}$ the coordinate system can be rotated independently, and for integration over $d \mathbf{k}_{2}$ we choose again orientation of $\mathbf{q}$ along the axis $z$, which leads to the spherical angle $\Theta_{2}$ resulting form the product, $\mathbf{k}_{2} \cdot \mathbf{q}=k_{2} q \cos \Theta_{2}$.

The expression for $\delta w$ attains thus the form,

$$
\begin{aligned}
& \delta w=\frac{e^{2} D_{0}^{2}}{(2 \pi)^{3} \hbar \varepsilon_{0}^{2}} \int d^{3} q \frac{\sin ^{2}(q a)}{q^{2} a^{2}} \frac{\cos \Theta}{q^{2}} \\
& \int_{0}^{\infty} d k_{2} k_{2}^{2} \int_{0}^{\pi} d \Theta_{2} \sin \Theta_{2} \int_{0}^{2 \pi} d \psi_{2} \frac{1}{\alpha+\beta} \frac{1}{2 \beta^{\prime} q k_{2}} \delta\left(\cos \Theta_{2}-\frac{k_{2}^{2}+\beta^{\prime} q^{2}-\gamma^{\prime}}{2 \beta^{\prime} q k_{2}}\right) .
\end{aligned}
$$

Integration over $d \Theta_{2}$ employs the Dirac delta. The relevant nonzero contribution (due to integration over $d \cos \Theta_{2}$ ) is conditioned by the inequality,

$$
-1<\frac{k_{2}^{2}+\beta^{\prime} q^{2}-\gamma^{\prime}}{2 \beta^{\prime} q k_{2}}<1
$$

which resolves itself to the condition,

$$
\left|\beta^{\prime} q-\sqrt{\gamma^{\prime}-\left(1-\beta^{\prime}\right) \beta^{\prime} q^{2}}\right|<k_{2}<\beta^{\prime} q+\sqrt{\gamma^{\prime}-\left(1-\beta^{\prime}\right) \beta^{\prime} q^{2}} .
$$

Thus,

and hence,

$$
\begin{aligned}
& \delta w=\frac{1}{(\alpha+\beta) 2 \beta^{\prime}} \frac{e^{2} D_{0}^{2}}{2 \pi \hbar \varepsilon_{0}^{2}} \int_{0}^{\sqrt{\frac{\gamma^{\prime}}{\beta^{\prime}\left(1-\beta^{\prime}\right)}}} d q q^{2} \frac{\sin ^{2} q a}{(q a)^{2}} \\
& \times \int_{0}^{\pi} d \Theta \sin \Theta \cos ^{2} \Theta \frac{1}{q^{3}} \int_{\mid \beta^{\prime} q-\sqrt{\gamma^{\prime}-\left(1-\beta^{\prime}\right) \beta^{\prime} q^{2} \mid}}^{\beta^{\prime} q+\sqrt{\gamma^{\prime}-\left(1-\beta^{\prime}\right) \beta^{\prime} q^{2}}} d k_{2} k_{2} .
\end{aligned}
$$

$$
\delta w=\frac{2}{3} \frac{\sqrt{\gamma}}{\alpha+\beta} \frac{e^{2} D_{0}^{2}}{2 \pi \hbar \varepsilon_{0}^{2}} \frac{1}{\zeta} \int_{0}^{\frac{1}{\zeta}} d x \frac{\sin ^{2}(x(a / \zeta))}{(x)^{2}(a / \zeta)^{2}} \sqrt{1-x)^{2}},
$$

where, $\zeta=\sqrt{\frac{\left(1-\beta^{\prime}\right) \beta^{\prime}}{\gamma^{\prime}}}$. Therefore,

$$
\begin{aligned}
& \delta w=\frac{4}{3} \frac{\mu^{2}\left(m_{n}^{*}+m_{p}^{*}\right) 2\left(\hbar \omega-E_{g}\right) e^{2} D_{0}^{2}}{\sqrt{m_{n}^{*} m_{p}^{*}} 2 \pi \hbar^{5} \varepsilon_{0}^{2}} \int_{0}^{1} d x \frac{\sin ^{2}(x a / \zeta)}{(x a / \zeta)^{2}} \sqrt{1-x^{2}} \\
& =\frac{4}{3} \frac{\mu^{2}}{\sqrt{m_{n}^{*} m_{p}^{*}}} \frac{e^{2} D_{0}^{2}}{2 \pi \hbar^{3} \varepsilon_{0}^{2}} \xi^{2} \int_{0}^{1} d x \frac{\sin ^{2}(x a \xi)}{(x a \xi)^{2}} \sqrt{1-x^{2}},
\end{aligned}
$$


where, $\xi=1 / \zeta=\frac{\sqrt{2\left(\hbar \omega-E_{g}\right)\left(m_{n}^{*}+m_{p}^{*}\right)}}{\hbar}$.

In limiting cases, we obtain finally,

$$
\delta w= \begin{cases}\frac{1}{3} \frac{\mu^{2}\left(m_{n}^{*}+m_{p}^{*}\right) 2\left(\hbar \omega-E_{g}\right) e^{2} D_{0}^{2}}{\sqrt{m_{n}^{*} m_{p}^{*} 2 \hbar^{5} \varepsilon_{0}^{2}}}, a \xi \ll 1, \\ \frac{4}{3} \frac{\mu^{2}\left(m_{n}^{*}+m_{p}^{*}\right) 2\left(\hbar \omega-E_{g}\right) e^{2} D_{0}^{2}}{\sqrt{m_{n}^{*} m_{p}^{*} 4 a \hbar^{*} \hbar^{5} \varepsilon_{0}^{2}}}, a \xi \gg 1,\end{cases}
$$

In the latter case the following approximation was applied,

$$
\int_{0}^{1} \frac{\sin ^{2}(x a \xi)}{(x a \xi)^{2}} \approx(\text { for } a \xi \gg 1) \frac{1}{a \xi} \int_{0}^{\infty} d(x a \xi) \frac{\sin ^{2}(x a \xi)}{(x a \xi)^{2}}=\frac{\pi}{2 a \xi}
$$

while in the former one, $\int_{0}^{1} d x \sqrt{1-x^{2}}=\pi / 4$.

The result must be multiplied by 4 (due to spin) and, in the case of half-space, divided by 4 . In the considered limiting cases we obtain thus,

$$
\delta w=\left\{\begin{array}{l}
\frac{1(4)}{3} \frac{\mu \sqrt{m_{n}^{*} m_{p}^{*}}\left(\hbar \omega-E_{g}\right) e^{2} D_{0}^{2}}{\hbar^{5} \varepsilon_{0}^{2}} \text { for } a \xi \ll 1, \\
\frac{1(4)}{3} \frac{\mu^{3 / 2} \sqrt{2} \sqrt{\hbar \omega-E_{g}} e^{2} D_{0}^{2}}{a \hbar^{4} \varepsilon_{0}^{2}} \text { for } a \xi \gg 1,
\end{array}\right.
$$

where (4) is addressed to the completely embedded nanosphere.

One can notice that the above formulae are quite distinct in comparison to the ordinary photo-effect. In the latter case, the perturbation is given by the vector potential in the kinematic momentum (for gauge, $\operatorname{div} \mathbf{A}=0$ ),

$$
\hat{H}=\frac{\left(-i \hbar \nabla-\frac{e}{c} \mathbf{A}(\mathbf{R}, t)\right)^{2}}{2 m^{*}} \simeq \frac{(-i \hbar \nabla))^{2}}{2 m^{*}}+\frac{i \hbar e}{m^{*} c} \mathbf{A}(\mathbf{R}, t) \cdot \nabla .
$$

For monochromatic plane wave, this perturbation has the form,

$$
\begin{aligned}
& w(\mathbf{R}, t)=\frac{i e \hbar}{c m^{*}}\left(\mathbf{A}_{0} \cdot \nabla\right) \cos (\omega t-\mathbf{k} \cdot \mathbf{R}+\alpha) \\
& =\frac{i e \hbar}{2 c m^{*}}\left(\mathbf{A}_{0} \cdot \nabla\right)\left(e^{i(\omega t-\mathbf{k} \cdot \mathbf{R}+\alpha)}+e^{-i(\omega t-\mathbf{k} \cdot \mathbf{R}+\alpha)}\right) .
\end{aligned}
$$

Because in this case, both states of band electrons and photon have the form of plane waves, the matrix element in the Fermi golden rule will be proportional to the Dirac delta with respect to the momentum sum. This expresses momentum conservation in translationally invariant system, what is a case for photon interacting with the semiconductor,

$$
\begin{aligned}
& <\mathbf{k}_{1}|w(\mathbf{R}, t)| \mathbf{k}_{2}>=\frac{i \hbar e}{2 c m^{*}(2 \pi)^{3}} \int d^{3} R e^{-i\left(\mathbf{k}_{1}+\mathbf{k}\right) \cdot \mathbf{R}}\left(\mathbf{A}_{0} \cdot \nabla\right) e^{i \mathbf{k}_{2} \cdot \mathbf{R}} \\
& =-\frac{e \hbar}{2 c m^{*}} \mathbf{A}_{0} \cdot \mathbf{k}_{2} \delta\left(\mathbf{k}_{1}+\mathbf{k}-\mathbf{k}_{2}\right) .
\end{aligned}
$$

Because of high value of photon velocity $c$, only vertical transitions, $\mathbf{k}_{1}=\mathbf{k}_{2}$, are admitted in the ordinary photo-effect.

Nevertheless, this is no case for nanosphere plasmon interacting in near-field regime with the semiconductor substrate. This system is not translationally invariant and indirect inter-band electron excitations are admitted resulting in enhancement of total transition probability. 
To compare with the ordinary photo-effect, let us recall the appropriate calculus upon the Fermi golden rule scheme,

$$
w\left(\mathbf{k}_{1}, \mathbf{k}_{2}\right)=\frac{\pi e^{2} \hbar}{2 c^{2} m^{* 2}} k_{2}^{2} A_{0}^{2} \cos ^{2} \Theta \delta^{2}\left(\mathbf{k}_{1}-\mathbf{k}_{2}\right) \delta\left(E_{1}-E_{2}+\hbar \omega\right),
$$

where, $\Theta$ is the angle between $\mathbf{k}_{2}$ i $\mathbf{A}_{0}$. One can use the following approximation in order to get rid the Dirac delta square,

$$
\delta^{2}\left(\mathbf{k}_{1}-\mathbf{k}_{2}\right)=\delta(0) \delta\left(\mathbf{k}_{1}-\mathbf{k}_{2}\right) \simeq \frac{V}{(2 \pi)^{3}} \delta\left(\mathbf{k}_{1}-\mathbf{k}_{2}\right),
$$

Similarly as was previously discussed, the normalization of probability must be performed. Thus we get,

$$
w\left(\mathbf{k}_{1}, \mathbf{k}_{2}\right)=\frac{\pi e^{2} \hbar}{2 c^{2} m^{* 2}} \frac{V}{(2 \pi)^{3}} k_{2}^{2} A_{0}^{2} \cos ^{2} \Theta \delta\left(\mathbf{k}_{1}-\mathbf{k}_{2}\right) \delta\left(E_{1}-E_{2}+\hbar \omega\right) .
$$

The integration over all states in both electron bands results in,

$$
\delta w=\int d^{3} k_{1} \int d^{3} k_{2} \frac{\pi e^{2} \hbar}{2 c^{2} m^{* 2}} \frac{V}{(2 \pi)^{3}} k_{2}^{2} A_{0}^{2} \cos ^{2} \Theta \delta\left(\mathbf{k}_{1}-\mathbf{k}_{2}\right) \delta\left(E_{1}-E_{2}+\hbar \omega\right),
$$

or

$$
\delta w=\int d^{3} k_{2} \frac{\pi e^{2} \hbar}{2 c^{2} m_{p}^{* 2}} \frac{V}{(2 \pi)^{3}} k_{2}^{2} A_{0}^{2} \cos ^{2} \Theta \delta\left(E_{1}\left(\mathbf{k}_{2}\right)-E_{2}\left(\mathbf{k}_{2}\right)+\hbar \omega\right),
$$

where the effective mass in the initial valence state is $m_{p}^{*}$ (moreover, $A_{0}=\frac{c}{\omega} E_{0}$ ). We thus obtain,

$$
\delta w=\frac{1}{12} \frac{e^{2} \hbar}{m_{p}^{*}} \frac{V}{\pi} \frac{E_{0}^{2}}{\omega^{2}} \frac{2^{3 / 2} \mu^{5 / 2}}{\hbar^{5}}\left(\hbar \omega-E_{g}\right)^{3 / 2} .
$$

One can rewrite it in the form,

$$
\delta w=\frac{4 \sqrt{2}}{3} \frac{\mu^{5 / 2} e^{2}}{m^{* 2} \omega \varepsilon_{0} \hbar^{3}}\left(\frac{\varepsilon_{0} E_{0}^{2} V}{8 \pi \hbar \omega}\right)\left(\hbar \omega-E_{g}\right)^{3 / 2},
$$

this expression should be multiplied by 4 (due to spin degeneracy, though for circular polarization of photons this factor is only 2 , due to angular momentum selection rules).

Taking into account, that the number of photons in the volume $V$ equals to, $\left(\frac{\varepsilon_{0} E_{0}^{2} V}{8 \pi \hbar \omega}\right)$, then the probability of single photon attenuation by the semiconductor per time unit, attains the form,

$$
q=\delta w\left(\frac{\varepsilon_{0} E_{0}^{2} V}{8 \pi \hbar \omega}\right)^{-1}=\frac{4(4) \sqrt{2}}{3} \frac{\mu^{5 / 2} e^{2}}{m^{* 2} \omega \varepsilon_{0} \hbar^{3}}\left(\hbar \omega-E_{g}\right)^{3 / 2},
$$

(factor (4) corresponds here to spin degeneration of band electrons).

In order to assess efficiency of the plasmon near-field coupling channel one can estimate the ratio of the probability of energy absorption in the semiconductor via mediation of surface plasmons (per single photon incident on the metallic nanospheres, $q_{m}$ ) to the energy attenuation in the semiconductor directly from a planar wave illumination (also per single photon, $q$ ). The ratio $\frac{q_{m}}{q}$ turns out to be of order of $10^{4} \frac{\beta 40}{H[n m]}$ (at a typical surface density 
of nanoparticles, $n_{s} \sim 10^{8} / \mathrm{cm}^{2}$ ), which (including the phenomenological factor $\beta$ [to account for proximity surface effects, not directly included], and $\mathrm{H}$-the semiconductor photo-active layer depth) is sufficient to explain the scale of the experimentally observed strong enhancement of absorption and emission rates. The strong enhancement of this transition probability is linked with allowance of momentum-non-conserved transitions, which is, however, reducing with the radius $a$ growth, according to formulae (54). For e.g., $a \sim 10-60 \mathrm{~nm}$, this gives reducing of the transition probability, which we have included by the effective phenomenological factor $\beta$ (fitted from the experiment, $\beta \simeq 28 \times 10^{-3}\left(\frac{50}{a[n m}\right)^{2}$ ) in the case of atomic limit, $a=0$ [11]. The enhancement of the near-field induced inter-band transition, in the case of large nanospheres, is, however, still significant as the reducing role of size-related quenching of transitions is partly compensated by $\sim a^{3}$ growth of the dipole amplitude of plasmon oscillations.

High efficiency (even if decreased with growing $a$ ) of the near-field energy transfer from surface plasmons to the semiconductor substrate is caused mainly by a contribution of all inter-band transitions, not restricted here to the direct (vertical) ones as for ordinary photo-effect, due to the absence of the momentum conservation constraints for the nanosystem. The strengthening of the probability transition due to all indirect inter-band paths of excitations in the semiconductor is probably responsible for the observed experimentally strong enhancement of light absorption and emission in diode systems mediated by surface plasmons in nanoparticle surface coverings [26, 27, 30, 31, 33, 34].

In the balanced state, when the incoming energy of light is transferred to the semiconductor via plasmon near-field coupling, we deal with the stationary solution of driven and damped oscillator. The driving force is the electric field of the incident planar wave, and the damping force is the near-field energy transfer described by the $\frac{1}{\tau}$ (calculated using formulae (54) in the manner as described in [13]). The resulting red-shifted resonance with simultaneously reduced amplitude allows for the accommodation to the balance of energy transfer to the semiconductor with incident photon energy. Within this model, the amplitude of resonant plasmon oscillations $D_{0}(\omega)$ is thus shaped by $f(\omega)=\frac{1}{\sqrt{\left(\omega_{1}^{2}-\omega^{2}\right)^{2}+4 \omega^{2} / \tau^{2}}}$. The extremum of red-shifted resonance is attained at $\omega_{m}=\omega_{1} \sqrt{1-2\left(\omega_{1} \tau\right)^{-2}}$ with corresponding amplitude $\sim$ $\tau /\left(2 \sqrt{\omega_{1}^{2}-\tau^{-2}}\right)$. This shift is proportional to $1 /\left(\omega_{1} \tau^{2}\right)$ and scales with nanosphere radius $a$ similarly (diminishes with decreasing $a$ ) as in the experimental observations [30] (note again that for scattering-induced $1 / \tau_{0}$ the dependence on $a$ is opposite [grows with decreasing $a$ ]).

In order to compare with the experiment we can estimate the photo-current in the case of a metallic modified surface. This photo-current is given by $I^{\prime}=|e| N\left(q+q_{m}\right) A$, where $N$ is the number of incident photons and $q$ and $q_{m}$ are the probabilities of single photon attenuation in the ordinary photo-effect [16] and in that one enhanced due to the presence of metallic nanospheres; $A=\frac{\tau_{f}^{n}}{t_{n}}+\frac{\tau_{f}^{p}}{t_{p}}$ is the amplification factor $\left(\tau_{f}^{n(p)}\right.$ is the annihilation time of both sign carriers, $t_{n(p)}$ is the drive time for carriers [the time of traversing the distance between electrodes]). From the above formulae, it follows that (here $I=I^{\prime}\left(q_{m}=0\right)$, i.e., the photo-current without metallic modifications)

$$
\frac{I^{\prime}}{I}=1+7.95 \cdot 10^{5} c_{0} \frac{m_{p}^{*}}{m_{n}^{*}}\left(\frac{2 a}{100[1 \mathrm{~nm}]} \sqrt{\frac{\hbar \omega_{1}[e V]}{x}\left(\frac{m_{p}^{*}}{m}+\frac{m_{n}^{*}}{m}\right)}\right)^{3} \phi(x),
$$




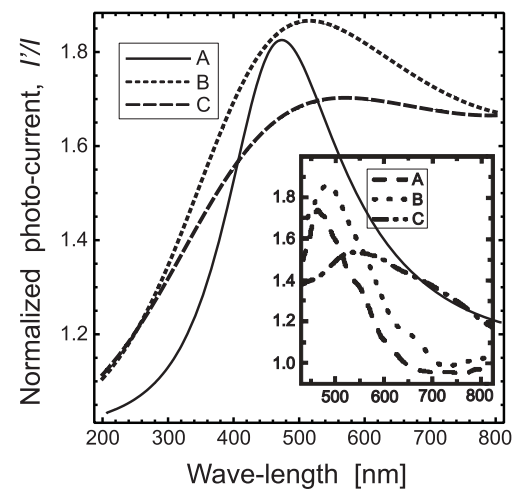

Figure 2. Dependence of the normalized photo-current $\frac{I^{\prime}}{I}(\lambda)$-comparison with the experimental data [30] for A: $a=25 \mathrm{~nm}$ (better fitting for $19 \mathrm{~nm}$ ), $n_{s}=6.6 \times 10^{8} 1 / \mathrm{cm}^{2}, \mathrm{~B}: a=40 \mathrm{~nm}, n_{s}=1.6 \times 10^{8} 1 / \mathrm{cm}^{2}$, C: $a=50 \mathrm{~nm}, n_{s}=0.8 \times 10^{8} 1 / \mathrm{cm}^{2}(H=3 \mu \mathrm{m})$

where $c_{0}=\frac{4 \pi a^{3}}{3} \beta \frac{n_{s}}{H}$, with $n_{s}$ as the surface density of metallic nanospheres, $H$ as the semiconductor layer depth, $\phi(x)=\frac{x^{2}}{\left(x^{2}-1\right)^{2}+4 x^{2} / x_{1}^{2}} \frac{1}{\sqrt{x-x_{g}}}, x=\omega / \omega_{1}, x_{1}=\tau \omega_{1}, x_{g}=$ $E_{g} /\left(\hbar \omega_{1}\right), \hbar \omega_{1}=2.72 \mathrm{eV}, m_{n(p)}$ as the effective mass of conduction band and valence band carriers (for $S i, m_{n}^{*}=0.19(0.98) m$ and $m_{p}^{*}=0.16(0.52) m$, for light (heavy) carriers, band gap $\left.E_{g}=1.14 \mathrm{eV}, \varepsilon=12\right), m$ as the bare electron mass.

The results are summarized in Fig. 2, and reproduce well the experimental behavior [30]. Both channels of photon absorption resulting in photo-current in the semiconductor sample are included, the direct ordinary photo-effect absorption with probability of transitions given by $q$ and the plasmon mediated absorption with probability $q_{m}$, respectively. Note also that some additional effects like reflection of the incident photons or destructive interference on metallic net would contribute and it was phenomenologically accounted for in the plasmon mediated channel by the experiment fitted factor $\beta$. These corrections are, however, rather not strong for the considered low densities of metallic coverings of order of $10^{8} / \mathrm{cm}^{2}$, and nanosphere sizes well lower than the resonant wave-length, though for larger concentrations and larger nanosphere sizes, would play a stronger reducing role $[19,32]$. The resonance threshold was accounted for by the damped resonance envelope function $\phi(x)$ in Eq. (66) including also semiconductor band-gap limit.

As indicated in Fig. 2, the relatively high value of $\frac{q_{m}}{q} \sim 10^{4} \frac{\beta 40}{H[n m]}$ enables a significant growth of the efficiency of the photo-energy transfer to the semiconductor, mediated by surface plasmons in nanoparticles deposited on the active layer, by increasing $\beta$ or reducing $H$ (at constant $n_{s}$ ). However, because of the fact that an enhancement of $\beta$ easily induces the overdamped regime, a greater perspective would be thus lowering $H$, the layer depth. The overall behavior of $I^{\prime} / I(\omega)=1+q_{m} / q$ calculated according to the relation (66), and depicted in Fig. 2, agrees quite well with the experimental observations [30], in the position, height and shape of the photo-current curves for distinct samples (the strongest enhancement is achieved for $a=40 \mathrm{~nm}$ ). 


\section{Nonlinear effect for undamped plasmon-polariton propagation along metallic nano-chain}

In the present chapter we report the RPA description using a semiclassical approach for a large metallic nanosphere (with radius of several tens $\mathrm{nm}$, and with $10^{5}-10^{7}$ electrons), in an all-analytical calculus version [11]. The electron liquid oscillations of compressional and translational type result in excitations inside the sphere and on its surface, respectively. They are referred to as volume and surface plasmons. Damping of plasmons due to electron scattering and due to radiation losses (accounted for via the Lorentz friction force) is included. The shift of the resonance frequency of dipole-type surface plasmons (only such plasmons are induced by homogeneous time-dependent electric field), due to damping phenomena, well fits with the experimental data for various nanosphere radii [15].

Collective dipole-type surface plasmon oscillations in the linear chain of metallic nanospheres were then analyzed and wave-type plasmon propagation along the chain was described $[13,36]$. A coupling in the near field regime between oscillating dipoles in neighboring nanospheres, together with retardation effects for energy irradiation, allowed for appearance of undamped propagation of plasmon waves (called plasmon-polaritons) along the chain in the experimentally realistic region of parameters (for separation of spheres in the chain and nanosphere radii). These effects are of a particular significance for plasmon arranged non-dissipative and sub-diffraction transport of converted light energy and information along metallic chains with promising expected applications in nano-electronics.

The undamped mode of plasmon-polaritons occurs, however, on the rim of stability of the linear approach [13]. It means that the zero damping rate separates the region with positive its value (corresponding to ordinary attenuation of plasmon-polaritons) and the region with negative damping rate (corresponding to instable plasmons-polariton modes). The latter indicates an unphysical behavior. i.e., the arte-fact of the linear approximation. In order to regularize this description the nonlinear corrections must be thus included. Inclusion of nonlinear corrections induced by the invariant form of the Lorentz friction results in such regularization. The instability region of linear approach is entirely covered by the region of undamped wave propagation with the amplitude accommodated to the nonlinearity scale and is independent of the initial condition (despite of its magnitude). This phenomenon, familiar in other nonlinear systems [2], seems to be of a particular significance for understanding of collective plasmon excitations with interesting possible applications.

\subsection{Nonlinear corrections to plasmon dynamics in phenomenological approach}

Even if the derivation of plasmon dynamics equation in the form of effective harmonic oscillator equation is rigid upon quantum approach of quasiclassical RPA method [11], the inclusion of plasmon attenuation of scattering type and of radiation losses type needs some phenomenological assumptions. They resolve themselves to extension of quantum RPA harmonic oscillator formulation to the damped oscillator equation form with attenuation described by heuristically assumed damping rates. It is proved $[11,14]$ that radiation losses in the case of the free far-field zone radiation (i.e., in the case of vacuum or dielectric surroundings of metallic nanosphere with oscillating plasmons) can be accounted for as the Lorentz friction [18]. Nevertheless, when in the near-field zone (closer than the wave length corresponding to plasmon frequency) the energy receiver (i.e., other charge system, like 
semiconductor with its band system or another metallic nanosphere, as in the chain) is located, the irradiation losses are dominated by energy transfer via this near-field zone coupling channel. Existence of charged system of energy receiver modifies retarded e-m potential of emitting system and this modifies the Lorentz friction formula, which was derived in the standard form for dielectric surroundings. The enhancement of radiation losses in the case when the nanoparticles with dipole Mie surface plasmons (excited by incident external light) are deposited on the semiconductor surface lies behind the observed PV efficiency growth in new generation of solar cells metallically modified [24, 25, 27, 30, 32, 34]. In this case the related attenuation rate can be also estimated by application of the Fermi golden rule to the semiconductor inter-band transitions induced by dipole near-field coupling with plasmons $[11,14]$, as was demonstrated above. As it was proved [11], the resulting attenuation rate scales with nano-sphere radius, $a$, in a different way in comparison to far-field radiation losses in dielectric medium, as is displayed by the formulae (53), additionally with a renormalization coefficient expressed in terms of the band system parameters [11]. One can expect the similar behavior in the case of the near-field coupling between nanospheres in the chain, but for the sake of effectiveness of modeling one can assume that related attenuation rate has the form as that for the Lorentz friction renormalized only by some coefficient phenomenologically assumed and accounting for a mentioned modification of e-m potential space distribution, caused by the receiver presence apart of the source.

Let us consider first a single metallic nanosphere with dipole type surface oscillations with the dipole,

$$
\boldsymbol{D}=e N_{e} a \boldsymbol{R},
$$

where $a$ is the nanosphere radius (of order of $10-50 \mathrm{~nm}$ ), $N_{e}$ is the number of electrons, $e$ is the electron charge. Oscillating dipole emits radiation and related energy losses can be expressed by the Lorentz friction (eventually renormalized by some phenomenological factor, as commented above). The general relativistic invariant formula for Lorentz friction force is as follows [18],

$$
\begin{aligned}
& g_{i}=\frac{2}{3} \frac{\left(e N_{e}\right)^{2}}{c}\left(\frac{d^{2} u_{i}}{d s^{2}}+u_{i} u_{k} \frac{d^{2} u_{k}}{d s^{2}}\right), \\
& d s=c d t \sqrt{1-\frac{v^{2}}{c^{2}}}, \\
& \boldsymbol{u}=\frac{\boldsymbol{v}}{c \sqrt{1-\frac{v^{2}}{c^{2}}}}, u_{4}=\frac{i}{\sqrt{1-\frac{v^{2}}{c^{2}}}}, \\
& \boldsymbol{v}=a \omega_{1} \dot{\boldsymbol{R}}, \dot{\boldsymbol{R}} \equiv \frac{1}{\omega_{1}} \frac{d \boldsymbol{R}}{d t},
\end{aligned}
$$

The latter expression slightly modifies the definition of dipole derivation in (68) as it incorporates plasmon frequency, for the sake of simplicity of further formulae. As the velocity can be expressed by the dipole derivation, the lowest order of power expansion of relativistic denominator gives the nonlinear corrections to the Lorentz friction, which can be accounted for in the formula for the Lorentz friction induced electric field,

$$
\boldsymbol{E}_{l}=\frac{2}{3} \frac{\sqrt{\epsilon}}{c^{3}}\left(e N_{e}\right) a \omega_{1}^{3}\left\{\dddot{\boldsymbol{R}}+\frac{\epsilon a^{2} \omega_{1}^{2}}{c^{2}} a\left(\frac{3}{2} \boldsymbol{R}^{(3)} \dot{\boldsymbol{R}}^{2}+3 \ddot{\boldsymbol{R}}(\dot{\boldsymbol{R}} \cdot \ddot{\boldsymbol{R}})+\dot{\boldsymbol{R}}(\dot{\boldsymbol{R}} \cdot \dddot{\boldsymbol{R}})\right)\right\},
$$

with nanosphere dipole plasmon frequency, $\omega_{1}=\frac{\omega p}{\sqrt{3 \epsilon}}$ (in a dielectric surroundings). In the above expression for the Lorentz friction induced electric field, the first term with the third order derivation of the dipole is the usual linear part of the Lorentz friction, while the next contribution (in parenthesis) is the lowest order nonlinear correction. 
In the framework of perturbation method of solution of dynamic equation of oscillatory type for the dipole, one can notice that in the zero order of perturbation (when dissipation is neglected), $\ddot{\boldsymbol{R}}+\boldsymbol{R}=0$, from which it follows that $\dddot{\boldsymbol{R}}=-\dot{\boldsymbol{R}}$. Thus one can simplify the formula (69), which leads to dynamical equation for the plasmon dipole including, in the first order of perturbation, the damping of plasmons due to scattering $\left(\frac{1}{\tau_{0}}\right)$ and due to radiation losses, i.e., due to the Lorentz friction (expressed by the rest of the right-hand-side of the equation (70) with the first term of linear contribution and with the next term of nonlinear type; the overall renormalization coefficient due to near-field coupling as commented above, is assumed here 1 for the sake of simplicity, but it could be changed in the final result),

$$
\ddot{\boldsymbol{R}}+\boldsymbol{R}=-\frac{2}{\tau_{0} \omega_{1}} \dot{\boldsymbol{R}}-\frac{2}{3}\left(\frac{\omega_{p} a}{\sqrt{3} c}\right)^{3} \dot{\boldsymbol{R}}+\frac{2}{3}\left(\frac{\omega_{p} a}{\sqrt{3} c}\right)^{5}\left\{-\frac{5}{2} \dot{\boldsymbol{R}}(\dot{\boldsymbol{R}} \cdot \dot{\boldsymbol{R}})+3 \boldsymbol{R}(\dot{\boldsymbol{R}} \cdot \boldsymbol{R})\right\} .
$$

The above equation is complicated in mathematical sense and advanced methods of solutions must be applied, as described in [2]. According to this asymptotical methods for solution of nonlinear differential equation (70), one can find the solution in the following form,

$$
\begin{aligned}
& R(t)=\frac{A_{0} e^{-\frac{t}{\tau}}}{\sqrt{1+\frac{9}{8} \gamma A_{0}^{2}\left(1-e^{-\frac{2 t}{\tau}}\right)}} \cos \left(\omega_{1} t+\Theta_{0}\right), R=R \hat{n}, \hat{n}=\frac{r}{r}, \\
& \frac{1}{\tau \omega_{1}}=\frac{1}{\tau_{0} \omega_{1}}+\frac{1}{3}\left(\frac{\omega_{p} a}{\sqrt{3} c}\right)^{3} \approx \frac{1}{3}\left(\frac{\omega_{p} a}{\sqrt{3} c}\right)^{3}, \\
& \gamma=\tau \omega_{1} \frac{1}{3}\left(\frac{\omega_{p} a}{\sqrt{3} c}\right)^{5} .
\end{aligned}
$$

In the formulae (71) both coefficients $\frac{1}{\tau \omega_{1}}$ and $\gamma$ can be renormalized eventually by the mentioned above phenomenological factor (still held here 1, for simplicity). From the form of equation (71) it follows that $\frac{1}{\tau \omega_{1}}$ is always positive. Note that the scattering term, $\frac{1}{\tau_{0}}=$ $\frac{v_{F}}{2 a}+\frac{C v_{F}}{2 \lambda_{B}}$, where $v_{F}$ is the Fermi velocity in metal material of the nanosphere, $\lambda_{B}$ is the bulk mean free path and $C$ is the constant of unity order [11,14]. This scattering term is negligible (for nanosphere radius beyond $10 \mathrm{~nm}$ ) in comparison with the linear contribution of the Lorentz friction, as it is demonstrated in Fig. (3). The nonlinear correction scale is given by $\gamma \approx 10^{-4}\left(\frac{a[n m]}{10}\right)^{2}$. As this coefficient is small, one can neglect the related contribution in the denominator for the dipole solution (71), which results in ordinary linear solution of damped oscillations, which means that the nonlinear corrections to the Lorentz friction have no significance in the case of plasmon oscillations of a single nano-sphere.

This situation changes, however, considerably in the case of collective plasmon excitation propagating along the metallic nano-chain, as it will be described in the following paragraph.

\subsection{Collective plasmon wave-type propagation along the nano-chain in the nonlinear regime}

In the case of the metallic nano-chain one has to take into account the mutual affecting of nanospheres in the chain. Assuming that in the sphere located in the point $\boldsymbol{r}$ we deal with the 


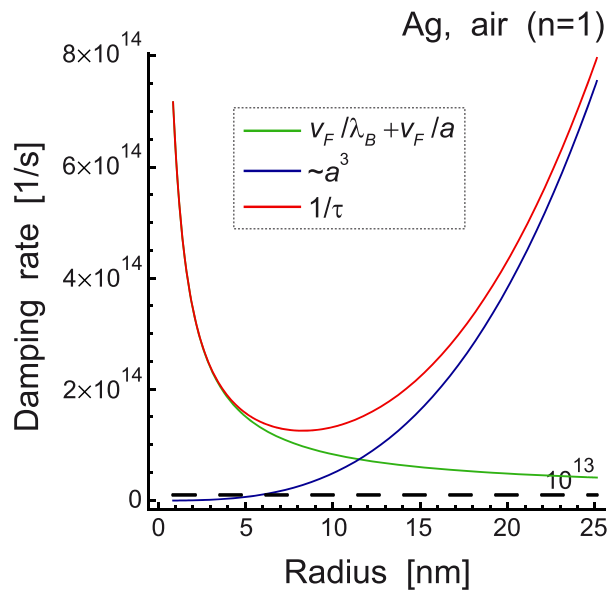

Figure 3. Contribution to the damping rate of surface plasmon oscillations in the nano-sphere versus the nano-sphere radius, including the scattering attenuation (green line) and the linear Lorentz friction damping (blue line); for radii greater than ca $10 \mathrm{~nm}$ the second channel dominates in the overall damping (red line)

dipole $\boldsymbol{D}$, then in the other place $\boldsymbol{r}_{0}$, the dipole type electric field attains the form as follows (including electro-magnetic retardation),

$$
\begin{aligned}
& \boldsymbol{E}\left(\boldsymbol{r}, \boldsymbol{r}_{0}, t\right)=\frac{1}{\epsilon r_{0}{ }^{3}}\left\{3 \boldsymbol{n}_{0}\left(\boldsymbol{n}_{0} \cdot \boldsymbol{D}\left(\boldsymbol{r}, t-\frac{r_{0}}{v}\right)\right)-\boldsymbol{D}\left(\boldsymbol{r}, t-\frac{r_{0}}{v}\right)\right\}, \\
& \boldsymbol{n}_{0}=\frac{\boldsymbol{r}_{0}}{r_{0}}, v=\frac{c}{\sqrt{\epsilon}} .
\end{aligned}
$$

This allows for writing of the dynamical equation for plasmons at each nanosphere of the chain which can be numbered by integer $l$ ( $d$ is here the separation between nanospheres in the chain). The first term of the right-hand-side in the following formula (73) describes the dipole type coupling between nanospheres [13] and the other two terms correspond to contribution due to plasmon attenuation (in the latter term the Lorentz friction caused electric field accounts also for nonlinear corrections). The index $\alpha$ enumerates polarizations, longitudinal and transversal ones with respect to the chain orientation.

$$
\begin{aligned}
& \ddot{R}_{\alpha}+R_{\alpha}(l d, t)=\sigma_{\alpha} \frac{a^{3}}{d^{3}} \sum_{m=-\infty, m \neq 1}^{\infty} \frac{R_{\alpha}\left(m d, t-\frac{d \mid l-m}{v}\right)}{|l-m|^{3}}-\frac{2}{\tau_{0} \omega_{1}} \dot{R}_{\alpha}(l d, t)+\frac{e}{m a \omega_{1}{ }^{2}} E_{\alpha}(l d, t), \\
& \sigma_{\alpha}=\left\{\begin{array}{l}
-1, \alpha=x, y \\
2, \alpha=z
\end{array}\right.
\end{aligned}
$$

The summation in the first term of the right-hand-side of the equation (73) can be explicitly performed in the manner as presented in [13], because it is the same as for the linear theory formulation. Similarly as in the linear theory framework, one can change to the Fourier picture taking advantage of the chain periodicity (in analogy to crystals with the reciprocal lattice), i.e.,

$$
\begin{aligned}
& R_{\alpha}(l d, t)=\tilde{R}_{\alpha}(k, t) e^{-i k l d}, \\
& 0 \leq k \leq \frac{2 \pi}{d}, \tilde{R}_{\alpha}(k) \sin \left(t \omega_{1}+\beta\right) .
\end{aligned}
$$


Thus the equation (73) can be rewritten in the following form (the Lorentz friction term was represented similarly as in equation (70)),

$$
\begin{aligned}
& \ddot{\tilde{R}}_{\alpha}(k, t)+\tilde{\omega}_{\alpha}^{2} \tilde{R}_{\alpha}(k, t)\left\{\frac{1}{\tau_{\alpha} \omega_{1}}+\frac{1}{3}\left(\frac{\omega_{p}}{\sqrt{3} c}\right)^{5}\left(\frac{5}{2}\left|\dot{\tilde{R}}_{\alpha}(k, t)\right|^{2}-3\left|\tilde{R}_{\alpha}(k, t)\right|^{2}\right)\right\}, \\
& \tilde{\omega}_{\alpha}^{2}=1-2 \sigma_{\alpha} \frac{a^{3}}{d^{3}} \cos (k d) \cos \left(\frac{d \omega_{1}}{v}\right), \\
& \frac{1}{\tau_{x, y} \omega_{1}}=\frac{1}{\tau_{0} \omega_{1}}+\frac{1}{4}\left(\frac{\omega_{1} d}{v}\right) \frac{a^{3}}{d^{3}}\left(\left(\frac{\omega_{1} d}{v}\right)^{2}-(k d-\pi)^{2}+\frac{\pi^{2}}{3}\right), \\
& \frac{1}{\tau_{z} \omega_{1}}=\frac{1}{\tau_{0} \omega_{1}}+\frac{1}{2}\left(\frac{\omega_{1} d}{v}\right) \frac{a^{3}}{d^{3}}\left(\left(\frac{\omega_{1} d}{v}\right)^{2}-(k d-\pi)^{2}+\frac{\pi^{2}}{3}\right),
\end{aligned}
$$

In the above formulae the remarkable property is linked with the expressions for the attenuation rate for both polarizations. Two last expressions in equation (75) give these damping rates explicitly and one can notice that they may change their signs depending on values for $d, a, k$. In Fig. (4) the region of negative value for damping rates is depicted (for both polarizations).

$\mathrm{Ag}, \mathrm{d} / \mathrm{a}=3$
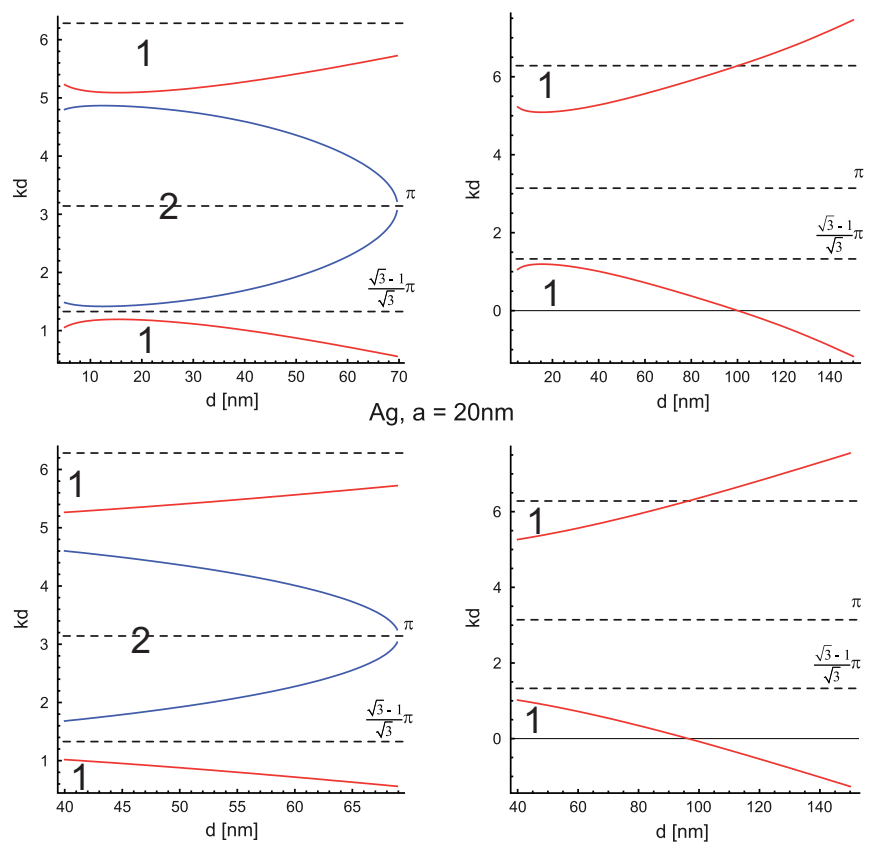

Figure 4. Regions for negative value of damping rates for plasmon-polaritons in the chain ( $1 \mathrm{for}$ longitudinal polarization modes and 2 for transversal ones, in the nolinear formulation framework; for the linear theory (red lines gives the position of vanishing damping rate for longitudinal modes of plasmon-polaritons and blue lines the same for transversal modes)

Applying the same methods for solution of the nonlinear equation (75) as in the former paragraph using the asymptotic methods [2], one can find the corresponding solutions for 
both regions with positive and negative damping rate, respectively,

$$
\begin{aligned}
& \text { for } \frac{1}{\tau_{\alpha} \omega_{1}}>0 \text {, } \\
& \tilde{R}_{\alpha}(k, t)=\frac{A_{\alpha 0} e^{-\frac{t}{\tau_{\alpha}}}}{\sqrt{1+\gamma_{\alpha} A_{\alpha 0}^{2}\left(1-e^{-\frac{2 t}{\tau_{\alpha}}}\right)}} \cos \left(\omega_{\alpha} t+\Theta_{0}\right) \rightarrow_{t \rightarrow \infty} 0, \\
& \gamma_{\alpha}=\left|\tau_{\alpha} \omega_{1}\right|\left(\frac{\omega_{1} a}{c}\right)^{3} \frac{1}{4}\left(\frac{5}{2} \tilde{\omega}_{\alpha}^{2}-1\right) \text {; } \\
& \text { for } \frac{1}{\tau_{\alpha} \omega_{1}}<0 \text {, } \\
& \tilde{R}_{\alpha}(k, t)=\frac{A_{\alpha 0} e^{\frac{t}{\left|\tau_{\alpha}\right|}}}{\sqrt{1+\gamma_{\alpha} A_{\alpha 0}^{2}\left(e^{\frac{2 t}{\tau_{\alpha} \mid}}-1\right)}} \cos \left(\omega_{\alpha} t+\Theta_{0}\right) \rightarrow_{t \rightarrow \infty} \frac{1}{\sqrt{\gamma_{\alpha}}} \cos \left(t \omega_{\alpha}+\Theta_{0}\right), \\
& D_{\alpha}=\frac{e N_{e} a}{\sqrt{\gamma_{\alpha}}} \frac{1}{2}\left\{e^{i\left(\omega_{\alpha} t-k l d\right)}+e^{-i\left(\omega_{\alpha}+k l d\right)}\right\} \text {. }
\end{aligned}
$$

From the above formulae it follows that for positive attenuation rate we deal with ordinary damped plasmon-polariton propagation, not strongly modified in comparison to linear theory (due to small value of the factor $\gamma$ ). Nevertheless, in the case of negative damping rate the solution behaves differently, i.e., in longer time scale this solution stabilizes on the constant amplitude value independently of initial conditions. This remarkable property characterizes undamped propagation of plasmon-polariton along the chain (if one turns back to dipole explicit form (67), then typical planar wave formula with constant amplitude describes this undamped mode, as written in the last equation of (76)). The region of negative damping corresponds thus, within the nonlinear approach, to undamped modes with the fixed amplitude. Let us note that the same region was linked with instability of the linear theory (which was, however, the arte-fact of the linear approach).

Finally, one can calculate the group velocity of the undamped plasmon-polariton mode in the following form,

$$
v_{\alpha}=\frac{d \omega_{\alpha}}{d k}=\omega_{1} d \frac{\sigma_{\text {alpha }} a^{3} \sin (k d) \cos \left(\frac{d \omega_{1}}{c}\right)}{d^{3} \sqrt{1-2 \sigma_{\alpha} \frac{a^{3}}{d^{3}} \cos (k d) \cos \left(\frac{d \omega_{1}}{c}\right)}} .
$$

From this formula it follows that the group velocity of the undamped wave type collective plasmon excitation (called plasmon-polariton) may attain different values depending on $a, d, k$.

Indicated above undamped mode of propagation of collective surface plasmons seems to match with experimentally observed long range propagation of plasmon excitations along the metallic nano-chain $[1,6,21,22,36]$. The constant and fixed value of the amplitude for these oscillations (76) are independent of initial conditions, which means that these excitations will be present in the system even if are excited by arbitrarily small fluctuations. Thus one can conclude that these are self-exciting modes which are always present in the system provided that radii of spheres and their separation in the chain have values for which attenuation rates (75) are negative.

\section{Conclusions}

We have demonstrated the practical utilization of RPA semiclassical description of plasmon oscillations in metallic nanospheres. The oscillatory form of dynamics both for volume and 
surface plasmons, rigorously described upon the RPA semiclassical limit fits well with the large nanosphere case, when the nanosphere radius is greater than $10 \mathrm{~nm}$ and lower than $60 \mathrm{~nm}$, (for $\mathrm{Au}, \mathrm{Ag}$ or $\mathrm{Cu}$ material), what is confirmed by experimental observations, on the other hand. The most important property of plasmons on such large nanospheres is the very strong e-m irradiation caused by these excitations, which results in quick damping of oscillations. The attenuation effects for plasmons were not, however, included into the quantum RPA model. Nevertheless, they could be included in a phanomenological manner, taking advantage of the oscillatory form of dynamic equations. Some information on plasmon damping can be taken from microscopic analyzes of small metallic clusters (especially made by LDA and TDLDA methods of numerical simulations employing Kohn-Sham equation). For larger nanospheres, these effects, mainly of scattering type (also Landau damping), are, however, not specially important as diminishing with radius growth as $\frac{1}{a}$. The irradiation effects overwhelming the energy losses in the case of large nanospheres can be grasped in terms of the Lorentz friction, which reduces the charge movement. This approach has been analyzed in the present paper. Two distinct situations were indicated, the first one-of the free radiation to far-field zone in dielectric/vacuum surroundings of single nanoparticle and the second one, when in the near-field zone of plasmons on the nanosphere, an additional charged system is located. This additional charge system of the e-m energy receiver strongly modifies the e-m potentials of the source and in this way modifies energy emission. In particular, the Lorentz friction is modified in this case in comparison to simple free emission to far-field zone. The e-m energy receiver located close to emitting nanosphere, could be semiconductor (as in the case of metallically modified solar cells) or other metallic nanospheres (as in the case of metallic nano-chain). Both these situations have been analyzed in this chapter.

The energy transfer from surface plasmon oscillations in metallic nanospheres deposited on photo-active surface of semiconductor to the substrate electron band system has been described upon the scheme of Fermi golden rule. The dependence of the effect with respect to the nanosphere dimension has been analyzed in details and the comparison with experimental data is given. We have elucidated the reason of the experimentally observed giant enhancement of PV efficiency caused by plasmon effect. Surface plasmons in metallic nanocomponents deposited on the semiconductor surface act as solar energy converters. The surface plasmons excited in the metallic nanospheres by incident photos couple with band electrons in the semiconductor substrate in the near-field regime. This dipole type coupling is more effective in comparison to the ordinary photo-effect coupling of electrons with incident photons, due to allowance of indirect inter-band transitions in the metallically locally modified systems. The metallic nanosphere coupled with substrate electrons in near-field zone of plasmon oscillations is not translational invariant system and thus the momentum conservation does not constrain quantum transitions in this case. This leads to strong enhancement of the inter-band transition probability, quenched, however, with the nanosphere radius growth, in the manner as presented in this paper.

With regard to collective plasmon fluctuations in ordered metallic systems, we have shown previously that along the infinite nano-chain the collective plasmon-polaritons can propagate (being collective surface plasmons coupled by e-m field in near-field zone), which at certain values of nanosphere radii and separation in the chain, appear as undamped modes. Simultaneously, the instability regions of linear theory of plasmon-polariton dynamics occur, which shows that the nonlinear corrections must be included. In this paper we have 
developed the nonlinear theory of collective plasmon-polariton dynamics along the chain, including nonlinear corrections to Lorentz friction force. Even though the related nonlinearity is small, it suffices to regularize the instable linear approach. As the most important observation, we noted the presence of undamped excitations (instead of those instable within the linear approach), which have fixed amplitude independently how small or large the initial conditions were. This excitations, typical for nonlinear systems, would have some practical significance, e.g., to enhance sensitivity of antennas with coverings by plasmon systems offering self-induced collective plasmon-polaritons in wide range of frequencies, which would be excited by even very small signal (the energy to attain the stable level of plasmon-polariton amplitude would be supplied, in this case, by an external auxiliary source).

\section{Acknowledgments}

Supported by NCN project No: 166719 Sonata 2

\section{Author details}

Jacak Witold

Institute of Physics, Wrocław University of Technology, Wyb. Wyspiańskiego 27, 50-370 Wrocław, Poland

\section{References}

[1] Barnes, W. L., Dereux, A. \& Ebbesen, T. W. [2003]. Nature 424: 824.

[2] Bogolubov, N. N. \& Mitropolskyj, J. A. [2005]. Asymptotical methods in theory of nonlinear oscillations, Nauka, Moscow.

[3] Bohren, C. F. \& Huffman, D. R. [1983]. Absorption and Scattering of Light by Small Particles, Wiley, New York.

[4] Brack, M. [1989]. Phys. Rev. B 39: 3533.

[5] Brack, M. [1993]. Rev. of Mod. Phys. 65: 667.

[6] Brongersma, M. L., Hartman, J. W. \& Atwater, H. A. [2000]. Phys. Rev. B 62: R16356.

[7] Ekardt, W. [1984]. Phys. Rev. Lett. 52: 1925.

[8] Ekardt, W. [1985]. Phys. Rev. B 31: 6360.

[9] Ekardt, W. [1986]. Phys. Rev. B 33: 8803.

[10] Garcia de Abajo, F. J. [2010]. Rev. Mod. Phys. 82: 209.

[11] Jacak, J., Krasnyj, J., Jacak, W., Gonczarek, R., A.Chepok \& Jacak, L. [2010]. Phys. Rev. B 82: 035418.

[12] Jacak, L., Krasnyj, J. \& Chepok, A. [2009]. Fiz. Nisk. Temp. 35: 491.

[13] Jacak, W., Krasnyj, J., Jacak, J., Chepok, A., Jacak, L., Donderowicz, W., Hu, D. \& Schaadt, D. [2010]. J. Appl. Phys. 108: 084304.

[14] Jacak, W., Krasnyj, J., Jacak, J., Donderowicz, W. \& Jacak, L. [2011]. J. Phys. D: Appl. Phys. 44: 055301.

[15] Jacak, W., Krasnyj, J., Jacak, J., Gonczarek, R., Chepok, A., Jacak, L., Hu, D. \& Schaadt, D. [2010]. J. Appl. Phys. 107: 124317.

[16] Kiriejew, P. S. [1969]. Physics of Semiconductors, PWN, Warsaw.

[17] Kresin, V. V. [1992]. Phys. Rep. 220: 1. 
[18] Landau, L. D. \& Lifshitz, E. M. [1973]. Field Theory, Nauka, Moscow.

[19] Losurdo, M., Giangregorio, M. M., Bianco, G. V., Sacchetti, A., Capezzuto, P. \& Bruno, G. [2009]. Solar Energy Mat. E Solar Cells 93: 1749.

[20] Maier, S. A. [2007]. Plasmonics: Fundamentals and Applications, Springer, Berlin.

[21] Maier, S. A., Kik, P. G. \& Atwater, H. A. [2003]. Phys. Rev. B 67: 205402.

[22] Markel, V. A. \& Sarychev, A. K. [2007]. Phys. Rev. B 75: 085426.

[23] Mie, G. [1908]. Ann. Phys. 25: 337.

[24] Morfa, A. J., Rowlen, K. L., Reilly, T. H., Romero, M. J. \& Lagemaat, J. [2008]. Appl. Phys. Lett. 92: 013504.

[25] Okamoto, K., Niki, I., Scherer, A., Narukawa, Y. \& Kawakami, Y. [2005]. Appl. Phys. Lett. 87: 071102.

[26] Okamoto, K., Niki, I., Shvartser, A., Narukawa, Y., Mukai, T. \& Scherer, A. [2004]. Nature Mat. 3: 601 .

[27] Pillai, S., Catchpole, K. R., Trupke, T., Zhang, G., Zhao, J. \& Green, M. A., [2006]. Appl. Phys. Lett. 88: 161102.

[28] Pines, D. [1999]. Elementary Excitations in Solids, ABP Perseus Books, Massachusetts.

[29] Pines, D. \& Bohm, D. [1952]. Phys. Rev. 92: 609.

[30] Schaadt, D. M., Feng, B. \& Yu, E. T. [2005]. Appl. Phys. Lett. 86: 063106.

[31] Stuart, H. R. \& Hall, D. G. [1998]. Appl. Phys. Lett. 73: 3815.

[32] Sundararajan, S. P., Grandy, N. K., Mirin, N. \& Halas, N. J. [2008]. Nano Lett. 8: 624.

[33] Wen, C., Ishikawa, K., Kishima, M. \& Yamada, K. [2000]. Sol. Cells 61: 339.

[34] Westphalen, M., Kreibig, U., Rostalski, J., Lüth, H. \& Meissner, D. [2003]. Sol. Energy Mater. Sol. Cells 61: 97.

[35] Yannouleas, C., Broglia, R. A., Brack, M. \& Bortignon, P. F. [1989]. Phys. Rev. Lett. 63: 255.

[36] Zayats, A. V., Smolyaninov, I. I. \& Maradudin, A. A. [2005]. Phys. Rep. 408: 131. 